Gregor Thüsing

\title{
Arbeitsrechtliche Vorlagen an den EuGH: Eine aktuelle Bestandsaufnahme
}

\section{Vom Sinn und Unsinn der Vorlagen an den EuGH}

Europarecht interpretiert authentisch nur der EuGH. Nach Art. 234 EG kann der nationale Instanzrichter beim EuGH anfragen, wenn er bei der Auslegung entscheidungserheblichen Europarechts zweifelt; der letztinstanzliche Richter muss in diesem Fall vorlegen. Deutsche Gerichte folgen dem bereitwillig. Sie führen die aktuelle Statistik an: 71 Verfahren im Jahr 2008 gegenüber z.B. nur 12 aufgrund Vorlage französischer Gerichte und 14 britische Verfahren. Der Unterschied liegt sicherlich nicht darin begründet, dass der deutsche Gesetzgeber besonders nachlässig in der Umsetzung wäre - denn in der Zahl der Vertragsverletzungsverfahren liegt Italien mit 17 neuen Verfahren im Jahr 2008 weit vorne. Deutschland steht hier auf den hinteren Rängen mit 10 Verfahren.

Warum dennoch die zahlreichen Vorlagen? Schaut man auf die jüngeren Anfragen im Bereich des Arbeitsrechts, so scheint es nicht falsch, diese Vorlagen nach dem Motiv der vorlegenden Gerichte zu ordnen. Die weitaus meisten Fälle zielen darauf ab, durch eine klare Antwort der Luxemburger Richter eine Rechtsunsicherheit auf nationaler Ebene zu beseitigen. Wenn man weiß, was das Europarecht will, lässt es sich einfacher europarechtskonform auslegen. Nah daran sind Vorlagen, bei denen ein Gericht zwar recht klare Vorstellungen davon hat, wie der Fall auf Grundlage der bisherigen Rechtsprechung des EuGH zu beurteilen ist. Das Ergebnis scheint jedoch unbillig und man will dem EuGH die Gelegenheit geben, zu widerrufen oder doch seine bisherige Rechtsprechung einzuschränken. Das Verfahren Ayse Süzen, ${ }^{1}$ das einige Misstöne der Entscheidung Christel Schmidt ${ }^{2}$ zu überwinden half, steht exemplarisch für diese Gruppe. Auch dies scheint mir ein sinnvoller Umgang mit dem EuGH zu sein, zielt er doch auf eine Weiterentwicklung seiner Rechtsprechung hin zu einem besseren Ergebnis. Nur wenn der EuGH angefragt wird, hat er die Gelegenheit, Fehlinterpretationen vorangegangener Rechtsprechung oder auch eigene Fehltritte zu korrigieren. Diese Chance sollte man ihm geben, sieht man denn die reelle Möglichkeit einer geänderten Rechtsprechung und zeigt sich die bisherige Rechtsprechung als allzu sperrig vor dem Hintergrund des nationalen Rechts.

Daneben stehen dann Vorlagen, die nicht auf eine Änderung der Rechtsprechung des EuGH, sondern der Rechtsprechung des BAG zielen. Man zielt auf Erfurt, wählt aber den Umweg über Luxemburg. Jüngeres Beispiel war das Verfahren Werhof. ${ }^{3}$ Der EuGH sollte das BAG zwingen, seine Rechtsprechung zur Gleichstellungsabrede aufzugeben. Der Schuss ging nach hinten los: Das BAG änderte zwischenzeitlich seine Rechtsprechung, der EuGH bestätigte jedoch die Europarechtskonformität der bisherigen Entscheidungslinie, und so wird das Urteil bereits dahingehend ausgelegt, dass die geän-

1 EuGH v. 11.3.1997 - Rs. C-13/95, NZA 1997, 433.

2 EuGH v. 14.4.1994 - Rs. C-392/92, NZA 1994, 545.

3 EuGH v. 9.3.2006 - Rs. C-499/04, NZA 2006, 376. 
derte Rechtsprechung der Erfurter Richter europarechtswidrig sei. Was Impetus zur Änderung sein wollte, wird nun Waffe in der Hand der Apologeten des status quo. ${ }^{4}$

Eine letzte Gruppe von Verfahren schließlich zielt auf den deutschen Gesetzgeber. Das deutsche Recht ist klar, die Rechtslage (scheinbar?) eindeutig, doch das Votum des EuGH soll zu einer Änderung des nationalen Rechts führen. Beispiel hierfür ist das unsägliche Mangold-Verfahren ${ }^{5}$ und wohl auch die Rechtssache Junk ${ }^{6}$ und auch das Verfahren Schultze-Hoff zur Unverfallbarkeit des Urlaubsanspruch auch bei Dauererkrankung. ${ }^{7}$ Diese Vorlagen sind unterschiedlich hilfreich. Ein Gang nach Luxemburg, der Rechtsunsicherheit reduzieren will, dient den Parteien des Rechtsstreits und der Allgemeinheit gleichermaßen. Eine Vorlage, die allein Rechtsunsicherheit schafft, dient niemandem. Wer vorlegt, sollte bedenken, ob seine Vorlage mehr Schaden als Nutzen anrichtet. Eben deshalb kann auch der Wille, das BAG durch den EuGH zu überzeugen, nicht der richtige Weg sein. Wenn die BAG-Rechtsprechung europarechtlich fragwürdig ist, dann soll das BAG vorlegen. Wenn aber ein ganzer Senat solche Zweifel nicht hegt, dann ist es vertretbar zu warten, bis Brüssel durch ein Vertragsverletzungsverfahren einschreitet. Wenn ein solches Verfahren indes nicht eingeleitet wird, scheint der Widerspruch zum Europarecht so groß nicht gewesen zu sein.

Arbeitsrechtlicher Sachverstand im Hinblick auf das deutsche Recht ist eher in Erfurt als in Luxemburg zu vermuten. Im Zweifel sollte bei der Entscheidung der Instanzgerichte über die Vorlage Rechtssicherheit vor europarechtlichem Nebel gehen, Verfahrensbeschleunigung vor Rechtsfortbildung in ungewisse Richtung. Dies gilt erst recht, wenn das deutsche Recht klar sagt, was es meint. Oft fehlt schon die Voraussetzung der Vorlage, wenn eine unmittelbare Wirkung ausscheidet und nicht - wie im Verfahren Mangold - gänzlich unerwartet vom EuGH gefunden wurde aufgrund von Rechtsquellen, an die das vorlegende Gericht selbst nicht gedacht hat. ${ }^{8}$ Solche Vorlagen mit rechtspolitischem Beigeschmack sollten wohl überlegt sein. Und noch mehr will überlegt sein, was dann das deutsche Gericht aus der Entscheidung Luxemburgs macht. Die Antwort in der Entscheidung des BAG vom 26.4.2006, ${ }^{9} \S 14$ Abs. 3 TzBfG ohne Vertrauensschutz rückwirkend unangewendet zu lassen, und ein ebenso strenges Vorgehen in der Umsetzung von Schultz-Hoff ${ }^{10}$ waren politisch unklug und juristisch nicht unangreifbar. Der Verzicht auf Vertrauensschutz mindert das Vertrauen in Europa und die Akzeptanz europäischen Rechts. Derjenige Arbeitgeber, der älteren Arbeitnehmern eine Chance gegeben hat und das tat, was der Gesetzgeber erhoffte, das er tun würde, ist nun der Dumme - mit der Begründung, die Europarechtskonformität sei schon immer umstritten gewesen. Soll der Bäckermeister künftig juristische Fachzeitschriften lesen, bevor er dem Gesetz vertrauen darf?

4 S. Nicolai, DB 2006, 670; hiergegen Thüsing, NZA 2006, 473.

5 EuGH v. 22.11.2005 - Rs. C-144/04, NZA 2005, 1345.

6 EuGH v. 27.1.2005 - Rs.C-188/03, NZA 2005, 213.

7 BAG v. 24.3.2009 - 9 AZR 983/07, NZA 2009, 538.

8 Zur Kritik auch auf europäischer Seite s. Generalanwalt Geelhoed, Schlussanträge Rs. C-13/05, Rn.56: "Aufgrund all dessen befürworte ich daher eine zurückhaltendere Auslegung und Anwendung der Richtlinie 2000/78, als sie der Gerichtshof in der Rechtssache Mangold vorgenommen hat".

9 BAG v. 26.4.2006 - 7 AZR 500/04, NZA 2006, 1162.

10 BAG v. 24.3.2009 - 9 AZR 983/07, NZA 2009, 538. 
Es gibt eine Pflicht der Gerichte, das Recht fortzubilden, ${ }^{11}$ und dazu gehört auch der Dialog zwischen nationalem und europäischem Gericht. Die systemstimmige Einbettung der Brüsseler Vorgaben liegt nicht allein in der Hand des Gesetzgebers, sondern auch beim Richter, der das Gesetz für den Einzelfall konkretisiert. Vorlagen, die Änderungen gesetzlicher Regelungen oder gefestigter nationaler Rechtsprechung vorbereiten wollen, sollten der letzten Instanz vorbehalten bleiben. Zu groß ist das Risiko unerwarteter Ergebnisse. Mag es de lege lata für diese Eingrenzung als verbindliche Regelung keine Grundlage geben, so wäre eine freiwillige Selbstbeschränkung der Instanzgerichte möglich und sinnvoll. Nicht alles muss nach Luxemburg.

\section{Aktuelle Vorlagen an den EuGH - Ein Überblick}

Dies vorweggeschickt ist es wichtig zu wissen, welche Vorlagen denn zurzeit beim EuGH liegen. Denn für das BAG ist die Vorlage an den EuGH das entscheidende Datum, ab dem es keinen Vertrauensschutz in den Fortbestand des bisherigen Verständnisses des nationalen Rechts geben kann. Das verwundert freilich, sind doch solche Vorlagen regelmäßig nicht veröffentlicht, oder doch nur in der jeweiligen Landessprache. Um dieses Vertrauen zu erschüttern seien also nachfolgend einige der wichtigeren, zurzeit anhängigen Verfahren in chronologischer Reihung kurz vorgestellt.

\section{Neues zur Altersgrenze? - VG Frankfurt v. 28. Mai 2008 - Colin Wolf gegen Stadt Frankfurt am Main (Rechtssache C-229/08)}

\section{Vorlagefragen:}

1. Besitzt der nationale Gesetzgeber für die Ausschöpfung der durch Art. 6 Abs. 1 RL 2000/78/EG eröffneten Spielräume durchgängig einen weiten Ermessens- und Gestaltungsspielraum oder wird dieser Spielraum jedenfalls dann auf das Notwendige eingeschränkt, wenn es um die Festlegung eines Höchstalters für die Einstellung im Hinblick auf eine Mindestdienstzeit bis zum Ruhestandseintritt entsprechend Art. 6 Abs. 1 Unterabsatz 2 lit. c RL 2000/78/EG geht?

2. Konkretisiert die Notwendigkeit in Art. 6 Abs. 1 Unterabsatz 2 lit. c RL 2000/78/ EG die Angemessenheit des in Art. 6 Abs. 1 Unterabsatz 1 RL 2000/78/EG genannten Mittels und schränkt so den Anwendungsbereich dieser allgemein gehaltenen Regelung ein?

3. a) Handelt es sich um ein legitimes Ziel im Rahmen von Art. 6 Abs. 1 Unterabsatz 1 RL 2000/78/EG, wenn ein Dienstherr durch ein Einstellungshöchstalter sein Interesse an einer möglichst langen aktiven Dienstzeit einzustellender Beamter verfolgt?

b) Ist die Umsetzung eines solchen Ziels schon unangemessen, wenn damit bewirkt wird, dass Beamte länger Dienst leisten als zum Erwerb der gesetzlich garantierten Mindestversorgung bei vorzeitigem Eintritt in den Ruhestand nach Ablauf von 5 Dienstjahren nötig?

c) Ist die Umsetzung eines solchen Ziels erst dann unangemessen, wenn damit bewirkt wird, dass Beamte länger Dienst leisten als zum Erdienen der gesetzlich garantierten

11 Dieterich, RdA 1993, 67. 
Mindestversorgung bei vorzeitigem Eintritt in den Ruhestand - derzeit 19,51 Jahre nötig?

4. a) Handelt es sich um ein i. S. d. Art. 6 Abs. 1 Unterabsatz 1 RL 2000/78/EG legitimes Ziel, durch ein möglichst geringes Einstellungshöchstalter die Zahl der insgesamt einzustellenden Beamten möglichst gering zu halten, um die Zahl der einzelfallbezogenen Leistungen wie Unfallfürsorge oder Krankenfürsorge (Beihilfen, auch für Familienangehörige) möglichst gering zu halten.

b) Welche Bedeutung kann insoweit dem Umstand zukommen, dass mit steigendem Lebensalter die Fürsorgeleistungen für Unfälle oder Beihilfen in Krankheitsfällen (auch Familienangehörige) höher ausfallen als bei jüngeren Beamten, sodass sich bei einer Einstellung lebensälterer Beamter oder Beamtinnen der insoweit zu leistende Aufwand insgesamt erhöhen könnte?

c) Müssen insoweit gesicherte Prognosen oder Statistiken vorliegen oder genügen allgemeine Wahrscheinlichkeitsannahmen?

5. a) Handelt es sich um ein i. S. d. Art. 6 Abs. 1 Unterabsatz 1 RL 2000/78/EG legitimes Ziel, wenn ein Dienstherr ein bestimmtes Einstellungshöchstalter zur Anwendung bringen will, um eine "ausgewogene Altersstruktur in der jeweiligen Laufbahn" zu gewährleisten?

b) Welchen Anforderungen müssen ggf. Erwägungen zur Gestaltung einer solchen Altersstruktur genügen, um die Voraussetzungen eines Rechtfertigungsgrundes (Angemessenheit und Erforderlichkeit, Notwendigkeit) zu erfüllen?

6. Handelt es sich um eine i. S. d. Art. 6 Abs. 1 Unterabsatz 1 RL 2000/78/EG legitime Erwägung, wenn der Dienstherr hinsichtlich eines Einstellungshöchstalters darauf verweist, bis zum Erreichen eines solchen Alters sei es regelmäßig möglich, die sachlichen Einstellungsvoraussetzungen für eine Ausbildung im mittleren Feuerwehrdienst in Gestalt einer entsprechenden Schulbildung und einer handwerklichen Ausbildung zu erwerben?

7. Nach welchen Kriterien ist zu beurteilen, ob eine Mindestdienstzeit bis zum Eintritt in den Ruhestand angemessen oder notwendig ist?

a) Lässt sich die Notwendigkeit einer Mindestdienstzeit ausschließlich als Äquivalent für den vom Dienstherrn allein finanzierten Erwerb einer Qualifikation beim Dienstherrn (Laufbahnbefähigung für den mittleren Feuerwehrdienst) rechtfertigen, um im Hinblick auf eine solche Qualifikation eine angemessene nachfolgende Dienstzeit bei diesem Dienstherrn sicher zu stellen, sodass die Ausbildungskosten vom Beamten auf diese Weise allmählich abgearbeitet werden?

b) Wie lange darf die Phase der auf die Ausbildungszeit nachfolgenden Dienstzeit höchstens sein? Darf sie 5 Jahre übersteigen, wenn ja unter welchen Voraussetzungen?

c) Kann die Angemessenheit oder Notwendigkeit einer Mindestdienstzeit unabhängig von Ziffer 7 Buchstabe a mit der Überlegung gerechtfertigt werden, dass bei Beamten, deren Ruhestandsversorgung der Dienstherr allein finanziert, die zu erwartende aktive Dienstzeit von der Einstellung bis zum voraussichtlichen Ruhestandseintritt ausreichen muss, um eine gesetzlich gewährleistete Mindestversorgung im Ruhestand durch eine Dienstzeit von derzeit 19,51 Jahren zu erdienen?

d) Ist umgekehrt die Ablehnung einer Einstellung nach Art. 6 Abs. 1 RL 2000/78/EG erst dann gerechtfertigt, wenn die Person mit einem Alter eingestellt würde, das bei 
voraussichtlichem Ruhestandseintritt dazu führen würde, dass die Mindestversorgung zu zahlen wäre, obwohl sie noch nicht erdient wäre?

8. a) Ist für die Beurteilung des Ruhestandseintritts nach Maßgabe von Art. 6 Abs. 1 Unterabsatz 2 lit. c RL 2000/78/EG auf die gesetzlich festgelegte Altersgrenze für den Übertritt in den Ruhestand mit anschließendem Bezug einer Pension abzustellen, oder muss auf das statistische Durchschnittsalter des Ruhestandseintritts einer bestimmten Beamten- oder Berufsgruppe abgestellt werden?

b) In welchem Ausmaß muss ggf. berücksichtigt werden, dass für einzelne Beamte der regelmäßige Übertritt in den Ruhestand um bis zu zwei Jahre hinausgeschoben werden kann? Führt dieser Umstand in entsprechendem Umfang zu einer Heraufsetzung des Einstellungshöchstalters?

9. Darf bei der Berechnung der Mindestdienstzeit im Rahmen von Art. 6 Abs. 1 RL 2000/78/EG die zunächst im Beamtenverhältnis zu absolvierende Ausbildung mitgerechnet werden? Ist insoweit von Bedeutung, ob die Ausbildungszeit beim Erwerb der Pension als ruhegehaltsfähige Dienstzeit in vollem Umfang zu berücksichtigen ist, oder ist die Ausbildungszeit aus der Zeitspanne herauszurechnen, für die ein Dienstherr eine Mindestdienstzeit nach Art. 6 Abs. 1 Unterabsatz 2 lit. c RL 2000/78/EG verlangen darf?

10. Sind die Regelungen in $\S 15$ Abs. 1 Satz 2, Abs. 3 Allgemeines Gleichbehandlungsgesetz mit Art. 17 RL 2000/78/EG vereinbar?

\section{a) Manchmal ist weniger mehr}

Das Gericht schießt mit Schrot. Zählt man alle Unterfragen mit, dann sind es 25 Antworten, die das VG Frankfurt vom EuGH erbittet. Es scheint mir ausgeschlossen, dass der EuGH auch nur ein Viertel davon beantworten wird. In der Vergangenheit hat er selten mehr als drei oder vier Fragen aus dem Konvolut der diversen Vorlagen aufgegriffen. Dies wird wohl auch hier gelten, auch weil viele der Fragen nicht vorlagefähig sind. Wann eine Benachteiligung wegen des Alters gerechtfertigt ist, ist vom nationalen Gericht zu entscheiden. Dies ist die zentrale Aussage der Entscheidung Palacios, ${ }^{12}$ die entsprechende Rechtsprechung zur mittelbaren Geschlechtsdiskriminierung, ${ }^{13}$ für die derselbe Rechtfertigungsmaßstab gilt, bestätigt hat. ${ }^{14}$ Es bedarf damit nicht des Umwegs über Luxemburg. Wäre das VG Frankfurt der Überzeugung, dass die in $\S 3$ Abs. 1 Nr. 1 der Verordnung über die Laufbahnen der Beamtinnen und Beamten des Einsatzdienstes der Berufsfeuerwehren ${ }^{15}$ des Landes Hessen genannte Höchstaltersgrenze nicht gerechtfertigt ist, dann liegt insoweit ein Verstoß gegen das AGG vor. Bundesrecht bricht Landesrecht. Das Gericht könnte durchentscheiden.

12 EuGH v. 16.10.2007 - Rs. C-411/05 - Palacios de la Villa, NZA 2007, 1219.

13 S. nur EuGH v. 23. 10. 2003 - Rs. C-4/02, Slg. I, 12575.

14 MünchKomm/Thüsing, §10 AGG Rnr. 10 m.w.N.

15 Feuerwehrlaufbahnverordnung - FeuerwLVO. 
b) Wie viel Generalisierung ist erlaubt?

Dennoch bleibt die Vorlage wertvoll, insbesondere die Frage 4 c). Inwieweit im Hinblick auf eine Rechtfertigung gesicherte Prognosen oder Statistiken vorliegen müssen oder allgemeine Wahrscheinlichkeitsannahmen genügen, wird eine der großen Fragen der Altersdiskriminierung sein. Allzu pauschale Verallgemeinerungen sind unverhältnismäßig. Ausländische Vorbilder sind hier sehr zurückhaltend, ${ }^{16}$ ebenso wie der EuGH in der Entscheidung Mangold. ${ }^{17}$ Generalanwältin Shaparton hat in ihren Anträgen zum Verfahren Bartsch ebenfalls eine eher strenge Linie vertreten. ${ }^{18}$ Der EuGH wird sich mit dieser Frage noch aufgrund einer Vorlage des SG Dortmund auseinandersetzen müssen, das die Altersgrenze der kassenärztlichen Zulassung vorgelegt hat: „Kann die gesetzliche Regelung einer Höchstaltersgrenze für die Zulassung zur Berufsausübung (hier: für die Tätigkeit als Vertragszahnärztin) im Sinne des Art. 6 der Richtlinie 2000/78/EG eine objektive und angemessene Maßnahme zum Schutz eines legitimen Zieles (hier: der Gesundheit der gesetzlich krankenversicherten Patienten) und ein zur Erreichung dieses Zieles angemessenes und erforderliches Mittel sein, wenn sie ausschließlich aus einer auf "allgemeine Lebenserfahrung" gestützten Annahme eines ab einem bestimmten Lebensalter eintretenden generellen Leistungsabfalls hergeleitet wird, ohne dass dabei dem individuellen Leistungsvermögen des konkret Betroffenen in irgendeiner Weise Rechnung getragen werden kann?". ${ }^{19}$ Die richtige Antwort fällt schwer. Gewisse Pauschalierungen sind möglich, ähnlich dem Maßstab beim allgemeinen Gleichbehandlungsgrundsatz: Eine Ungleichbehandlung ist zulässig, wenn ein Hilfskriterium zu einer Gruppenbildung verwandt wird, die ihrerseits auf eine Unterscheidung abzielt, die durch sachliche Gründe gerechtfertigt ist. Dies entspricht allgemeinen Regeln auch zur Einordnung gesetzlicher Differenzierungen. Erforderlich ist allein, dass sich durch die Unterscheidung nach den Hilfskriterien hinreichend sicher das eigentliche Ziel fördern lässt und - das ist entscheidend! - der Aufwand, eine feingliedrigere Abgrenzung vorzunehmen, unverhältnismäßig wäre. Ebenso wie also die Geschäftsfähigkeit anders als die Deliktsfähigkeit nicht in Ansehung des Einzelfalls bestimmt wird, sondern vergröbernd zwei Gruppen gebildet werden, bei denen eine hinreichende Verstandesreife vermutet oder nicht vermutet wird, kann es richtig sein, dass der Arbeitgeber nicht nach der körperlichen Konstitution im Einzelfall, sondern nach dem Alter differenziert. Man spricht von statistical discrimination. ${ }^{20}$

16 S. zum US-Recht: "reasonable factors other than age" (RFOA) ist die affirmative defense under 29 USC §623(f)(1). Hierzu demächst $v$. Hoff, Das Verbot der Altersdiskriminierung im Blickwinkel der ökonomischen Analyse des Rechts, 2009.

17 EuGH v. 22.11.2005 - C-144/04, NZA 2005, 1345.

18 Anträge v. 22. Mai 2008 Rs. C-427/06.

19 SG Dortmund v. 24. Juli 2008 - Dr. Domenica Petersen gegen Berufungsausschuss für Zahnärzte für den Bezirk Westfalen-Lippe (Rechtssache C-341/08), 1. Vorlagefrage.

20 S. Thüsing, RdA 2003, 257; Ausführlich demnächst v. Hoff, Das Verbot der Altersdiskriminierung im Blickwinkel der ökomischen Analyse des Rechts, 2009; zum allgemeinen Gleichbehandlungsgrundsatz s. HWK/Thüsing, § 611 BGB Rnr. 206. 
c) Höchstalter zur Einstellung in anderen Fällen

Wie ist es nun mit anderen Fällen? § 10 Nr. 3 AGG lässt die Festlegung eines Höchstalters für die Einstellung zu. Dem liegt die Überlegung zugrunde, dass bei älteren Beschäftigten, deren Rentenalter bereits absehbar ist, einer aufwendigen Einarbeitung am Arbeitsplatz auch eine betriebswirtschaftlich sinnvolle Mindestdauer einer produktiven Arbeitsleistung gegenüberstehen muss. ${ }^{21}$ Auch hier gilt jedoch das Gebot der Verhältnismäßigkeit, das solchen Beschränkungen engere Grenzen zieht, als sie in der Praxis oftmals zu finden sind. Wenn der Personalfluss so hoch ist, dass ein Großteil der Arbeitnehmer das Unternehmen jenseits der Einstellungsgrenze vor Erreichen des Ruhestands verlassen, dann greift das Argument ebenso wenig, wie wenn jüngere Arbeitnehmer in entsprechender Zeit zu einem erheblichen Teil den Arbeitgeber wechseln: Wenn nur wenige Arbeitnehmer tatsächlich zehn Jahre im Unternehmen bleiben, dann können 55-jährige Bewerber nicht mit dem Argument zurückgewiesen werden, eine aufwendige Einarbeitung sei nicht sinnvoll.

Möglicher Maßstab kann die bisherige Rechtsprechung zur Rückzahlung von Fortund Ausbildungskosten sein. ${ }^{22}$ Die zulässige Bindungsdauer steht danach in Abhängigkeit zur Länge der Fortbildungszeit. Sind auch letztlich die Einzelfallumstände entscheidend, so hat die Rechtsprechung gewisse Richtlinien zur Beurteilung eines angemessenen Verhältnisses zwischen Fortbildungsmaßnahme und Bindungsdauer herausgearbeitet. Im Einzelnen gilt Folgendes: Eine bis zu zwei Monaten dauernde Fortbildungsmaßnahme lässt eine Bindung von einem $\mathrm{Jahr} \mathrm{zu},{ }^{23}$ bei drei bis vier Monaten sind es schon zwei Jahre ${ }^{24}$ und einer Fortbildungsdauer von sechs bis zwölf Monaten vermag eine Bindungsdauer von drei Jahren zu rechtfertigen. ${ }^{25}$ Eine fünfjährige Bindungsdauer setzt eine mehr als zwei Jahre andauernde Fortbildungsmaßnahme voraus. ${ }^{26}$ Ein starres Schema ist das nicht, vielmehr gelten diese Grundsätze nur für den Regelfall. Im Einzelfall kann auch bei kürzerer Dauer der Fortbildung eine verhältnismäßig lange Bindung gerechtfertigt sein, wenn etwa der Arbeitgeber erhebliche Mittel aufwendet und die Teilnahme an der Fortbildung dem Arbeitnehmer besondere Vorteile bringt. Umgekehrt kann auch bei längerer Dauer der Fortbildung nur eine verhältnismäßig kurze Bindung gerechtfertigt sein. Das kann etwa dann der Fall sein, wenn der Arbeitgeber nur verhältnismäßig wenig Mittel aufwendet und die Teilnahme an der Fortbildung dem Arbeitnehmer nur geringe Vorteile bringt. ${ }^{27}$ Hier zeigt sich, dass der Rechtsprechung zur Bindungsdauer die Erwägung zugrunde liegt, dass die Dauer der Fortbildung zwar ein starkes Indiz für die Qualität der erworbenen Qualifikation ist und daher in besonderem Maße bei der Interessenabwägung berücksichtigt werden muss, der Qualifikati-

21 BT-Drucks. 16/1780 S. 36.

22 Ausf. HWK/Thüsing, § 611 BGB Rnr. 460 ff.

23 BAG v. 15.12.1993 - 5 AZR 279/93, NZA 1994, 835.

24 BAG v. 6.9.1995 - 5 AZR 241/94, NZA 1996, 314.

25 BAG v. 11.4.1984 - 5 AZR 430/82, NZA 1984, 288; BAG v. 23.4.1986 - 5 AZR 159/85, NZA 1986, 741

26 BAG v. 8.5.1974 - 5 AZR 359/73, NJW 1974, 2151; BAG v. 12.12.1979 - 5 AZR 1056/77, AP Nr. 4 zu $§ 611$ BGB Ausbildungsbeihilfe.

27 BAG v. 6.9.1995 - 5 AZR 241/94, NZA 1996, 314. 
onsgrad des Arbeitnehmers aber letztlich auch durch andere Umstände geprägt werden kann.

Bei der Ausdeutung des $\S 10$ Nr. 3 AGG sind diese Zeiten als Mindestrahmen anzunehmen, der Arbeitgeber wird jedoch deutlich darüber hinausgehen können, schon weil der Ruhestand das sichere Ausscheiden aus dem Betrieb bedeutet, nach Ablauf der Bindungsfrist jedoch der Arbeitnehmer durchaus noch weiterbeschäftigt werden kann und idR auch wird. Bei Zeiten jenseits von fünf Jahren wird man jedoch vorsichtig sein müssen. Je höherwertig die Position ist, desto eher wird man einen Ausschluss rentennaher Jahrgänge begründen können.

Wird ein Mitarbeiter zum Führungskräftenachwuchs gesucht, dann kann auch ein sehr junges Höchstalter gerechtfertigt sein. Maßgeblich ist, welche Zeit nach den regelmäßigen Karrierewegen des Unternehmens erforderlich wäre, um die angestrebte Führungsposition zu erreichen, und welche Zeit dann noch angemessen ist, diese Position im Unternehmen ausführen zu können. Generell gilt, dass Altersgrenzen eher akzeptabel sind, wo sie als weiche Zielvorgaben mit der Möglichkeit zur Einzelabwägung formuliert werden, als harte Ausschlusskriterien, die auch bei geringfügiger Überschreitung einen Bewerber aus dem Auswahlpool ausschließen (,Der Bewerber sollte Anfang 40 sein“ ist also vorzugswürdig gegenüber „Wir suchen einen Bewerber bis Alter 45 Jahre").

Eine mittelbare Diskriminierung wegen des Alters, die einen ähnlichen Effekt wie die Festsetzung von Höchst- und Mindestalter haben kann, ist das Erfordernis einer bestimmten Anzahl von Jahren Berufserfahrung. Als Mindestqualifikation kann sie gerechtfertigt sein durch die Erfordernisse der Tätigkeit und auch zur Vermeidung von überqualifizierten Bewerbern kann sie als Höchstrahmen erlaubt sein („,Wir suchen Bewerber von drei bis sechs Jahren Berufserfahrung"). Hinsichtlich der Vermeidung von Überqualifikationen dürfte jedoch ein strengerer Maßstab angebracht sein als im Hinblick auf eine Mindestdauer von Berufsjahren. Die Gefahr der Umgehung des Verbots der Altersdiskriminierung ist offensichtlich.

\section{Diskriminierung Teilzeitbeschäftigter - Vorabentscheidungsersuchen des Corte d'appello di Roma (Italien) vom 12.9.2008 - Istituto nazionale della previdenza sociale (INPS) / Tiziana Bruno und Massimo Pettini (Rs. C-395/08)}

\section{Vorlagefragen:}

Ist die Regelung des italienischen Staates (der oben genannte Art. 7 Abs. 1 des Gesetzes Nr. 638/83), die dazu führt, dass Zeiträume, in denen bei vertikaler Teilzeitarbeit nicht gearbeitet wird, nicht als Beitragszeiten für den Erwerb des Rentenanspruchs berücksichtigt werden, mit der Richtlinie 97/81/EG und insbesondere mit Paragraph 4 der Rahmenvereinbarung im Anhang zu dieser Richtlinie über den Grundsatz der Nichtdiskriminierung vereinbar?

Ist die oben angeführte nationale Regelung mit der Richtlinie vereinbar, insbesondere mit Paragraph 1 der Rahmenvereinbarung in Anhang zu dieser Richtlinie, der vorsieht, dass die nationale Regelung die Entwicklung der Teilzeitarbeit fördern soll, mit Paragraph 4 und mit Paragraph 5 der oben angeführten Rahmenvereinbarung, der die Mitgliedstaaten verpflichtet, Hindernisse rechtlicher Natur, die Teilzeitarbeitsmöglichkei- 
ten beschränken, zu beseitigen, vereinbar, wobei es außer Zweifel steht, dass die Nichtberücksichtigung von nicht gearbeiteten Wochen für die Rentenansprüche eine bedeutende Hemmschwelle für die Aufnahme von - vertikaler - Teilzeitarbeit darstellt?

Kann sich Paragraph 4 der oben angeführten Rahmenvereinbarung über den Grundsatz der Nichtdiskriminierung auch auf den Bereich der verschiedenen Teilzeitvertragsarten erstrecken, wenn man bedenkt, dass auf der Grundlage der nationalen Regelung bei horizontaler Teilzeitarbeit, d. h. bei gleichmäßig auf das Kalenderjahr verteilten Arbeitsstundenanzahl, im Gegensatz zu vertikaler Teilzeitarbeit alle Wochen des Kalenderjahres berücksichtigt werden?

\section{a) Die Vorlage als solche}

Die Vorlage bezieht sich auf italienisches Recht, und zwar auf Art. 7 Abs. 1 des Gesetzes Nr. 638/83: Wird Teilzeitarbeit im Blockmodell geleistet, dann erwirbt der Arbeitnehmer für die Zeiten der Freistellung keinen Rentenanspruch.

Aus deutscher Sicht ist die Vorlage erklärungsbedürftig. Sie zielt auf eine Ungleichbehandlung durch staatliches Gesetz, nicht durch den Arbeitgeber. Dieses Gesetz mag nun richtlinienwidrig sein, aber dennoch ist eine unmittelbare Wirkung nicht gegeben. Eine europarechtskonforme Auslegung ist geboten - eine europarechtskonforme Beugung ist unzulässig. Der italienische Gesetzgeber hat aber klar gesagt, was er meint. Nach Maßstäben deutscher Dogmatik wäre damit die Vorlage unzulässig, weil unerheblich: Selbst wenn die Norm europarechtswidrig wäre, könnte dem Arbeitnehmer der Anspruch nicht zugesprochen werden. ${ }^{28}$ Der italienische Jurist - wie die meisten romanischen Juristen - ist hier freilich großzügiger. Die strenge Trennung zwischen unmittelbarer Wirkung (,efficacia diretta“) und richtlinienkonformer Auslegung (,,interpretazione conforme") ist ihm weitgehend unbekannt. Eine europarechtskonforme Auslegung gleichsam „,contra legem“ wird oftmals gar nicht erst problematisiert. ${ }^{29}$ Dies wurde auch deutlich an den diesbezüglich ganz unbefangenen Anträgen des Generalanwalts Colomer in der Rechtssache Pfeiffer, ${ }^{30}$ die nur mühsam durch erneute Anträge und ein nachfolgendes, sehr viel präziseres Urteil eingefangen werden konnten.

Ist die Vorlage damit auf der Grundlage romanischer Gesetzesinterpretation wohl nicht unerheblich, so scheint sie dennoch unnötig. Der EuGH hat in der Vergangenheit stets die Frage des sachlichen Grundes dem nationalen Gericht zur Entscheidung über-

28 Zur parallelen Problematik der Quelle-Entscheidung des EuGH (es ging materiell um ein Problem aus dem Kaufrecht, nämlich die Verpflichtung des Käufers zum Wertersatz im Falle der Nacherfüllung nach $\S \S 346,439$ IV BGB) s. zuletzt Pfeiffer, NJW 2009, 412.

29 S. aber Teresi, Direttive comunitarie inattuate e interpretazione conforme: dalla «interpositio legislatoris» all'applicazione giudiziaria, in Nuove aut., 1994, 71. Einen aktuellen guten Überblick bietet Acocella, Interpretazione conforme al diritto comunitario ed efficienza economica: il principio di concorrenza, abrufbar http://users.unimi.it/gruppodipisa2008/relazioni/acocella.pdf.

30 Rs. C-397/01 bis C-403/01; erste Anträge vom 6.5.2003. In den zweiten Schlussanträgen vom 27.4.2004 wurde die Frage dann problematisiert und zutreffend als ,eines der komplexesten Dilemmata des Gemeinschaftsrechts" bezeichnet. 
antwortet. ${ }^{31}$ Das italienische Gericht hätte also auch hier selber entscheiden können und wird es wohl auch müssen.

\section{b) Benachteiligung von Teilgruppen und mittelbare Diskriminierung}

Der Kern der Frage lässt sich abstrahieren: Der Staat benachteiligte nicht alle Teilzeitbeschäftigte gegenüber Vollzeitbeschäftigten, sondern nur solche, die einen gewissen Zeitraum überhaupt nicht arbeiteten. Nach der Rechtsprechung des BAG liegt hier klar eine Benachteiligung wegen der Teilzeitbeschäftigung vor, auch ohne, dass man dem EuGH vorgelegt hätte, denn: „Die unterschiedliche Behandlung einer Gruppe teilzeitbeschäftigter Arbeitnehmer gegenüber den vollzeitbeschäftigten Arbeitnehmern entfällt nicht dadurch, dass der Arbeitgeber eine andere Gruppe teilzeitbeschäftigter Arbeitnehmer nicht diskriminiert". Das hatte das BAG bereits verschiedentlich so festgestellt, und die Literatur ist dem weitgehend gefolgt. ${ }^{32}$ Es lohnt freilich hierüber nachzudenken. Die Frage begegnet dem Arbeitsrechtler ähnlich bei allen anderen Diskriminierungsverboten: Ist die Benachteiligung wegen eines Merkmals, das nur mit einem verbotenen Unterscheidungsmerkmal verbunden sein kann, aber nicht stets mit ihm verbunden ist, eine unmittelbare Diskriminierung oder nur eine mittelbare Diskriminierung? Der erkennende Senat hat sich hier für eine unmittelbare Diskriminierung entschieden, ebenso wie sich das BAG bei der Benachteiligung wegen einer Schwangerschaft in Übereinstimmung mit der Rechtsprechung des EuGH für unmittelbare Geschlechtsdiskriminierung entschieden hat. ${ }^{33}$ Schaut man genauer hin, dann kommen Zweifel, ob dies richtig ist. Unmittelbare Diskriminierung definiert sich dadurch, dass am verbotenen Unterscheidungsmerkmal selbst angeknüpft wird. Das ist aber hier nicht der Fall: Bloße Teilzeitbeschäftigung allein reicht nicht zum Anspruchsausschluss. Mittelbare Diskriminierung kennzeichnet sich dadurch, dass nach einem Merkmal unterschieden wird, das zumeist mit dem verbotenen Unterscheidungsmerkmal verbunden ist, aber nicht immer. Es kann also bei beiden Gruppen vorkommen, wenn auch überproportional bei einer von beiden. Auch dies trifft es hier nicht ganz, denn das Hilfsmerkmal kann ausschließlich bei einer der beiden Gruppen vorkommen, nicht überwiegend. Die Unterscheidung liegt also dogmatisch zwischen beiden Diskriminierungsformen, und eindeutige Argumente, sie der einen oder anderen Gruppe zuzuordnen, gibt es nicht. Im US-amerikanischen Schrifttum wird diese Frage unter dem Stichwort der sex-plus bzw. religionplus discrimination erörtert: gemeint ist eine Ungleichbehandlung wegen des Geschlechts und eines weiteren Merkmals (etwa: weiblich und schwanger bei der Benachteiligung wegen Schwangerschaft, durch den Pregnancy Discrimination Act 1978 ausdrücklich im US-amerikanischen Recht [im Widerspruch zu vorangegangener Recht-

31 Zumindest im Hinblick auf die mittelbare Geschlechtsdiskriminierung von Teilzeitbeschäftigten, s. bereits EuGH v. 13.5.1986 - Rs C-170/84, Slg. I 1986, 1620.

32 S. zuletzt BAG v. 24.9.2003 - 10 AZR 675/02, NZA 2004, 611; davor BAG v. 12.6.19965 AZR 960/94, NZA 1997, 191; BAG v. 1.11.1995 - 5 AZR 84/94, NZA 1996, 813; BAG v. 26.9.2001 - 10 AZR 714/00, AP Nr. 1 zu § 4 TzBfG; s. auch Thüsing, in: Annuß/Thüsing, $\S 4$ TzBfG Rn. 24 m.w.N..

33 S. EuGH v. 4.10.2001 - Rs. C-109/00, DB 2001, 2451 mit Anmerkung Thüsing; auch Art. 2 76/207/EG seit der Änderung durch Richtlinie 2002/73/EG. 
sprechung] wie unmittelbare Geschlechtsdiskriminierung behandelt) bzw. wegen der Religion und eines weiteren Merkmals (katholisch und Jesuit bei der Benennung des Dekans einer vom Orden getragenen Hochschule). ${ }^{34}$ Man ist sich hier nicht sicher, welche Antwort richtig ist, und diese Unsicherheit kann der deutschen Rechtsprechung bewusst gemacht werden.

Die Frage dürfte freilich bei der Teilzeit nicht allzu bedeutend sein, denn auch die mittelbare Teilzeitdiskriminierung dürfte durch $\S 4$ Abs. 1 TzBfG verboten $\operatorname{sein}^{35}$ und der Rechtfertigungsmaßstab für beide Diskriminierungsformen liegt sehr nahe beieinander; beides mal ist es der sachliche Grund, mag auch dieser Begriff bei der mittelbaren Diskriminierung in der Praxis der Gerichte einige Pegelstriche großzügiger interpretiert werden als bei der unmittelbaren Diskriminierung. ${ }^{36}$ Bei anderen Merkmalen ist die Unterscheidung jedoch oftmals streitentscheidend, etwa beim Merkmal der Religion nach dem AGG. Wenn die Benachteiligung wegen eines islamischen Kopftuchs eine unmittelbare Diskriminierung wegen der Religion ist (islamisch und kopftuchtragend), dann bedarf es gemäß $\S 8$ AGG wesentlicher und entscheidender beruflicher Voraussetzungen zur Rechtfertigung; wenn es eine mittelbare Diskriminierung ist, dann reicht es, dass der Arbeitgeber darlegen kann, dass zwar dem Anschein nach neutrale Vorschriften, Kriterien oder Verfahren Personen mit einer bestimmten Religion gegenüber anderen Personen in besonderer Weise benachteiligen, diese Vorschriften, Kriterien oder Verfahren jedoch durch ein rechtmäßiges Ziel sachlich gerechtfertigt sind und die Mittel zur Erreichung dieses Ziels angemessen und erforderlich sind. ${ }^{37}$ Der Kopftuchfall könnte darüber hinaus ganz parallel auch unter das Verbot der unmittelbaren oder mittelbaren Geschlechtsdiskriminierung subsumiert werden, denn nur Frauen tragen ein islamisches Kopftuch, wenn auch nicht alle. Wäre also die in der vorliegenden Vorlagefrage aufgeworfene Problematik auf alle Diskriminierungsverbote zu übertragen, dann hätte das BAG die Kündigung der kopftuchtragenden Verkäuferin wohl schon an $\S 611$ a BGB, heute $\S \S 1,7$ Abs. 1 AGG scheitern lassen müssen. ${ }^{38}$

\section{c) Ausblick}

Das BAG hat seit einiger Zeit keine Fragen der Teilzeitdiskriminierung vorgelegt. Die jüngsten Entscheidungen überzeugen auch ohne Gang nach Luxemburg. ${ }^{39}$ Der EuGH hat soweit ersichtlich keine anderen Vorlagen zur Teilzeit anhängig. Die letzte Entscheidung in den Rs. Michaeler und Subito stieß auf wohlwollendes Echo. ${ }^{40}$ Es bleibt

34 Pime v. Loyola University of Chicago, 803 F.2 d 351 - 7th Cir. 1986.

35 Hierfür Däubler, in: Kittner/Däubler/Zwanziger, KSchR, § 4 TzBfG Rnr. 42; Schüren, MünchArbR, § 161, Rnr. 56; Wank, Anm. SAE 1994, 195, 196; Beduhn, AuR 1996, 485, 488; Coester, Anm. SAE 1995, 339, 342, 343. Dagegen MünchKomm-BGB/Müller-Glöge, § 4 TzBfG, Rnr. 16.

36 S. hierzu Thüsing, in: Annuß/Thüsing, § 4 TzBfG, Rnr. 18.

37 S. also in diesem Licht BAG v. 10.12.2002 - 2 AZR 472/01, AP Nr. 44 zu § 1 KSchG 1969 Verhaltensbedingte Kündigung, mit Anm. Adam; hierzu auch Thüsing, NJW 2003, 405.

38 S. auch Thüsing, Arbeitsrechtlicher Diskriminierungsschutz, 2007, S.99.

39 BAG v. 16.9.2008 - 9 AZR 781/07, ZTR 2009, 32; BAG v. 24.9.2008 - 10 AZR 634/07, ArbuR 2009, 590.

40 EuGH v. 24.4.2008 - C-55/07/ und C-56/07,,NZA 2008, 579. 
abzuwarten, ob der EuGH auch bei der vorliegenden Frage eine ähnliche glückliche Hand hat.

\section{Grenzen des Befristungsrechts - Vorabentscheidungsersuchen des Tribunale di} Trani (Italien). eingereicht am 6. März 2009-Francesca Sorge/Poste Italiane SpA ${ }^{41}$

\section{Vorlagefragen:}

Ist Paragraph 8 der durch die Richtlinie 1999/70/EG durchgeführten Rahmenvereinbarung dahin auszulegen, dass sie einer innerstaatlichen Regelung wie der in den Art. 1 und 11 des DL Nr. 368/2001 enthaltenen entgegensteht, durch die in Umsetzung der Richtlinie 1999/70/EG des Rates vom 28. Juni 1999 zu der EGB-UNICE-CEEPRahmenvereinbarung über befristete Arbeitsverträge ${ }^{1}$ Art. 1 Abs. 2 Buchst. b des Gesetzes Nr. 230/1962 aufgehoben wurde (nach welcher letzteren Bestimmung "die Befristung des Vertrags" zulässig war, "wenn die Anstellung erfolgt[e], um abwesende Arbeitnehmer zu vertreten, die Anspruch auf Erhaltung ihres Arbeitsplatzes [hatten], vorausgesetzt, im befristeten Arbeitsvertrag wurde der Name des vertretenen Arbeitnehmers und der Grund für seine Vertretung angeführt") und durch eine Bestimmung ersetzt wurde, die keine solchen Konkretisierungsverpflichtungen mehr vorsieht?

Sollte Frage $1 \mathrm{zu}$ bejahen sein: Hat das nationale Gericht die insofern gemeinschaftsrechtswidrige innerstaatliche Regelung unangewendet zu lassen?

\section{a) Die Vorlage}

Wieder eine italienische Vorlage. Es geht um Decreto Legislativo 6 settembre 2001, n. $368,{ }^{42}$ der italienischen Regelung zur Umsetzung der Befristungsrichtlinie. Weil einige - aber durchaus nicht alle - Elemente des Dekrets für den italienischen Arbeitnehmer ungünstiger sind als die vorangegangene Regelung, stellt das Gericht die Frage nach der Vereinbarkeit mit dem Absenkungsverbot des $\S 8$ Abs. 3 der RV-Befristung: „Die Umsetzung dieser Vereinbarung darf nicht als Rechtfertigung für die Senkung des allgemeinen Niveaus des Arbeitnehmerschutzes in dem von dieser Vereinbarung erfassten Bereich dienen“. Und wenn denn die Regelung unvereinbar ist: Was ist die Folge?

\section{b) Verwandte Fragen: Reichweite der Absenkungsverbote}

Die Frage stellt sich ganz allgemein und mutet bekannt an. Solche europarechtlichen Absenkungsverbote gibt es in vielen arbeitsrechtlichen Richtlinien. ${ }^{43}$ Ihre europarechtliche Zulässigkeit war immer streitig, geht es hier doch nicht mehr um die Harmonisierung von Mindestarbeitsbedingungen, sondern um deren Optimierung.

Auch für das deutsche Recht wurde diese Frage bereits im Befristungsrecht diskutiert. Dies galt nicht nur für $\S 14$ Abs. 3 TzBfG - Altersbefristung -, sondern auch für $\S 14$

41 Rs. C-98/09.

42 Gazzetta Ufficiale n. 235 del 9 ottobre 2001.

43 Z.B. Art. 8 Abs. 2 RL 2000/78/EG, Art. 23 RL 2003/88/EG. 
Abs. 2 TzBfG. Weil der hierdurch vermittelte Schutz hinter dem der Rechtsprechung vor Erlass des BeschFG 1985 zurückbleibe, liege hierin eine Absenkung des allgemeinen Schutzniveaus, für welche die Richtlinie nach $\S 8$ Abs. 3 RV-Befristung keinen Anlass geben darf. Das BeschFG und die bereits hier eingeräumte Möglichkeit zur Befristung ohne sachlichen Grund könne nicht berücksichtigt werden, da es als zeitlich befristetes Gesetz nicht geeignet sei, das allgemeine Schutzniveau zu bestimmen. ${ }^{44}$ Das war nicht überzeugend, ${ }^{45}$ und noch sehr viel deutlicher hat der EuGH zu $§ 14$ Abs. 3 TzBfG in der Rechtssache Mangold Stellung genommen: Es kommt darauf an, ob die Umsetzung die Rechtfertigung der Herabsenkung des Schutzes darstellen soll oder vielleicht nur ihr Anlass ist. ${ }^{46}$ Findet der Gesetzgeber andere sachliche Gründe für die Zurücknahme von Arbeitnehmerschutz, dann darf er sich auf diese auch berufen. Ähnlich argumentierte schon Generalanwalt Tizzano in seinen ansonsten schwierigen Schlussanträgen: „Was den Zweck der Bestimmung betrifft, bekräftige ich noch einmal, dass diese verhindern will, dass der einzelstaatliche Gesetzgeber die Richtlinie 1999/70 als Rechtfertigung für einen Abbau des den Arbeitnehmern gewährten Schutzes benutzt, indem er die Richtlinie für Regelungen verantwortlich macht, die das Ergebnis seiner eigenen unabhängigen gesetzgeberischen Entscheidung sind. “47 Deutlicher noch hat es aber Generalanwältin Kokott in ihren Schlussanträgen im Verfahren Angelidaki ${ }^{48}$ unter Berufung auf Mangold formuliert: „Erstens ist eine Verminderung des den Arbeitnehmern im Hinblick auf befristete Arbeitsverträge garantierten Schutzes nicht als solche durch die Rahmenvereinbarung verboten; eine derartige Verminderung bleibt vielmehr zulässig, wenn sie in keinem Zusammenhang mit der Umsetzung dieser Rahmenvereinbarung steht... Zweitens bezieht sich das Verschlechterungsverbot des Paragrafen 8 Nr. 3 der Rahmenvereinbarung allein auf eine Senkung des allgemeinen Niveaus des Arbeitnehmerschutzes im Hinblick auf befristete Beschäftigung. Der Abschaffung oder Abschwächung einer spezifischen Maßnahme des Arbeitnehmerschutzes steht das Verschlechterungsverbot also nicht entgegen, es sei denn, dadurch würde sich das Schutzniveau für befristet Beschäftigte insgesamt verringern“. Gemessen an dieser einschränkenden Interpretation des Absenkungsverbots muss die Antwort des Gerichtshofs wohl eher verneinend ausfallen.

c) Verdrängende unmittelbare Wirkung von Richtlinien?

Spannender noch ist die zweite Frage, wenn denn eine Richtlinienkonformität nicht gegeben ist. Es geht um die unmittelbare Wirkung von Richtlinien. Eine positive unmittelbare Wirkung in Horizontalverhältnissen, die dazu führt, dass eine Richtlinie als solche wie nationales Recht oder eine EG-Verordnung (Art. 249 Abs. 2 EG) Pflichten

44 Schmalenberg, NZA 2000, 1043, 1044; Däubler, ZIP 2000, 1961, 1967.

45 S. Thüsing/Lambrich, BB 2002, 829.

46 EuGH v. 22.12.2005 - Rs. C-144/04, NZA 2005, 1345 Rnr. 44 ff..

47 Anträge v. 30. 6. 2005 - Rs. C-144/04.

48 Anträge v. 4.12.2008 - Rs. C-378/07, Rnr. 68 f.. 
für Private begründet, hat der EuGH in ständiger Rechtsprechung abgelehnt. ${ }^{49}$ Eine negative unmittelbare Wirkung oder Ausschlusswirkung führt demgegenüber lediglich dazu, dass nationales Recht, das gegen eine Richtlinie verstößt, unanwendbar ist. Ist sie anzuerkennen? Die jüngste Stellungnahme aus Luxemburg entstammt hierzu den Anträgen des Generalanwalts Bot im Verfahren Kücükdevici. Obwohl dieser sehr weitgehende Vorstellungen hin zu einer unmittelbaren Wirkung hat, referiert er die bisherige Rechtsprechung zutreffend: „Das dritte Korrektiv [der fehlenden unmittelbaren Wirkung von Richtlinien unter Privaten] schließlich liegt in der „Abkoppelung“ der Möglichkeit der Berufung auf die Richtlinie von der horizontalen unmittelbaren Wirkung der Richtlinien, um das entgegenstehende nationale Recht auszuschließen, und zwar auch in einem Rechtsstreit zwischen Einzelnen. Diese Lösung läuft darauf hinaus, dass die Richtlinien zwar nicht an die Stelle des fehlenden oder säumigen nationalen Rechts treten können, um unmittelbar Verpflichtungen für einen Einzelnen zu begründen, dass aber wenigstens die Berufung auf sie möglich ist, um das entgegenstehende nationale Recht auszuschließen, so dass das nationale Gericht nur das von den richtlinienwidrigen Bestimmungen befreite nationale Recht für seine Entscheidung in einem Rechtsstreit zwischen Einzelnen anwendet. Diese Abkopplung der Möglichkeit der Berufung auf die Ausschlusswirkung der Richtlinien von der „ersetzenden“ unmittelbaren Wirkung der Richtlinien ist vom Gerichtshof jedoch niemals allgemein und ausdrücklich bestätigt worden. Die Bedeutung dieses dritten Korrektivs ist somit bisher sehr beschränkt geblieben.“. In der Tat: In der Vergangenheit haben sich schon Generalanwälte für eine negative unmittelbare Wirkung in Horizontalverhältnissen ausgesprochen. ${ }^{50}$ Auch ein Teil des Schrifttums befürwortet eine solche Richtlinienwirkung. ${ }^{51}$ Der EuGH hat eine solche Wirkung bisher nicht anerkannt. Die häufig angeführten Entscheidungen CIA Security $^{52}$ und Unilever ${ }^{53}$ lassen sich nicht zwingend als Anerkennung einer horizontalen unmittelbaren Wirkung verstehen. Vielmehr lassen sich die Fälle auf vertikale Konstellationen zurückführen. ${ }^{54}$ Es sind nun also zwei Verfahren, in denen der EuGH Gelegenheit hat, seine Rechtsprechung zur unmittelbaren Wirkung zu präzisieren oder zu revidieren.

49 EuGH v. 14.7.1994 - Rs. C-91/92 - Faccini Dori, Slg. 2004, I-3325 Rn 24; v. 7.3.1996 Rs. C-192/94 - El Corte Inglés, Slg. 1996, I-1281 Rnr. 17; v. 5.10.2004 - Rs. C-397-403/01 - Pfeiffer, NZA 2004, 1145.

50 Vgl. Schlussanträge von GA Saggio vom 16.12.1999 in der Rs. C-240-244/98 - Oceano Slg. 2000, I-4941 Rn 30 und GA Colomer vom 27.4.2004 in der Rs. C-397-403/01, Rnr. 29 ff - Pfeiffer - NZA 2004, 1145.

51 Herrmann, Richtlinienumsetzung durch die Rechtsprechung, 2003, S. 78 ff. m.w.N. auch aus dem englischsprachigen Schrifttum.

52 EuGH v. 30.4.1996 - Rs. C-194/94, Slg. 1996, I-2201.

53 EuGH v. 26.9.2000 - Rs. C-443/98, Slg. 2000, I-7535.

54 Gundel, EuZW 2001, 143, 147 f.; zum Ganzen auch von Medem, Kündigungsschutz und AGG, 2008, S. 204 ff. 
4. Geschlechtsdiskriminierung oder nicht? - Vorabentscheidungsersuchen des Tribunal Superior de Justicia de Galicia (Spanien), eingereicht am 19. März 2009 - Pedro Manuel Roca Álvarez/Sesa Start España E.T.T. S.A. ${ }^{55}$

Vorlagefrage:

Verstößt ein nationales Gesetz (konkret Art. 37 Abs. 4 des Arbeitnehmerstatuts), das das Recht auf eine bezahlte Arbeitsbefreiung für Stillzeiten, die in einer Verkürzung der täglichen Arbeitszeit um eine halbe Stunde oder in einer Arbeitsbefreiung von einer Stunde, die in zwei Abschnitte aufgeteilt werden kann, besteht, freiwilligen Charakter hat und vom Unternehmer zu vergüten ist, bis das Kind neun Monate alt ist, ausschließlich den in einem Lohn- oder Gehaltsverhältnis beschäftigten Müttern, nicht aber den in einem Lohn- oder Gehaltsverhältnis beschäftigten Vätern zuerkennt, gegen den Grundsatz der Gleichbehandlung, der jede Diskriminierung aus Gründen des Geschlechts verbietet und in Art. 13 des Vertrags, in der Richtlinie 76/207/EWG ${ }^{1}$ des Rates vom 9. Februar 1976 zur Verwirklichung des Grundsatzes der Gleichbehandlung von Männern und Frauen hinsichtlich des Zugangs zur Beschäftigung, zur Berufsbildung und zum beruflichen Aufstieg sowie in Bezug auf die Arbeitsbedingungen sowie in der Richtlinie 2002/732, durch die die genannte Richtlinie geändert wurde, verankert ist?

\section{a) Die Vorlage des Appelationsgerichts Gallicien}

Wieder mal eine Vorlage aus dem Bereich des Diskriminierungsrechts. Es geht um Art. 37 Abs. 4 Estatuto de los Trabajadores, dem zentralen spanischen Arbeitsgesetz: „Las trabajadoras, por lactancia de un hijo menor de nueve meses, tendrán derecho a una hora de ausencia del trabajo, que podrán dividir en dos fracciones. La mujer, por su voluntad, podrá sustituir este derecho por una reducción de la jornada normal en media hora con la misma finalidad. Este permiso podrá ser disfrutado indistintamente por la madre o el padre en caso de que ambos trabajen.“ Zu Deutsch etwa: „Arbeitnehmerinnen haben, wenn sie ein Kind von unter neun Monaten stillen, das Recht auf eine Stunde Pause. Diese kann in zwei Teile unterteilt werden. Die Frau hat ebenso das Recht, anstatt der Stunde Pause mit demselben Zweck ihre Tagesarbeitszeit um eine halbe Stunde zu verkürzen. Dieses Recht kann vom Vater oder von der Mutter in Anspruch genommen werden, soweit beide arbeitstätig sind“".

Die Vorschrift existiert seit dem 10. März 1980, geschaffen durch das ley 8/1980, 10 de marzo, del Estatuto de los Trabajores, die sich ihrerseits an älteren Vorschriften orientierte. Geändert wurde die Regelung durch real decreto legislativo 1/1995, 24 de marzo und letztmalig durch ley orgánica 3/2007, 22 de marzo. Ihr Inhalt hat prima facie nichts Anstößiges: Nur Frauen bekommen Stillpausen. ${ }^{56}$ Dass Männer die nicht bekommen überrascht auf den ersten Blick nicht allzu sehr: Wie wollten sie die Brust geben? Doch ein Fläschchen geben kann auch der Mann, und wenn beide Eltern arbeiten soll dementsprechend ja auch Frau oder Mann zur Arbeitsreduzierung berechtigt sein. Zwei Fragen treten damit in den Blick: Wann ist allgemein eine Ungleichbehandlung

55 Rs C-104/09.

$56 \mathrm{Zu}$ vergleichbaren deutschen Regelungen schon in der Weimarer Zeit s. Hueck/Nipperdey, Arbeitsrecht I, 1931, S. 470. 
zwischen den Geschlechtern erlaubt und wie wird das Gericht die konkrete Frage beantworten. $\mathrm{Zu}$ beidem einige kurze Sätze.

\section{b) Zulässige Ungleichbehandlung von Männern und Frauen}

Nicht jede Ungleichbehandlung wegen des Geschlechts ist verboten. Sie ist durch Art. 2 Abs. 6 der RL 76/207/EWG erlaubt, wenn das Geschlecht ,, aufgrund der Art einer bestimmten beruflichen Tätigkeit oder der Bedingungen ihrer Ausübung eine wesentliche und entscheidende berufliche Anforderung darstellt, sofern es sich um einen rechtmäßigen Zweck und eine angemessene Anforderung handelt". Die Differenzierung nach dem Geschlecht ist damit zumindest zulässig, wenn nur Männer oder Frauen die betreffende Tätigkeit ausführen können. Diese tatsächliche Notwendigkeit bilden die Fälle biologischer Notwendigkeit, für die beispielhaft die deutsche Kommentarliteratur gerade die Amme zählt. ${ }^{57}$ Hierzu gehört auch die Fallgruppe der Authentizitätswahrung: ${ }^{58}$ Damenmode kann authentisch nur von Damen vorgeführt werden, und: „A male playboy bunny would not be the same thing". 59

Zur rechtlichen Notwendigkeit gehören alle Fälle, in denen ein Gesetz die Beschäftigung des jeweils anderen Geschlechts verbietet. ${ }^{60}$ Hier kann kein Verstoß gegen das AGG - oder sein spanisches Pendant ${ }^{61}$ gegeben sein, weil es der vollständigen Umsetzung der dem Diskriminierungsverbot zugrunde liegenden Richtlinien entgegensteht. Solange der deutsche Gesetzgeber aber nicht tätig wird, um ein eventuelles Umsetzungsdefizit zu beheben, kann sich der Arbeitnehmer - wo er nicht staatlich Bediensteter ist und wo nicht das Verbot der Geschlechtsdiskriminierung beim Entgelt nach Art. 141 EG-Vertrag greift - nicht unmittelbar auf das Europäische Recht berufen, da dies eine horizontale verpflichtende Wirkung von Privaten bedeuten würde, die nach gefestigter Rechtsprechung des EuGH ausgeschlossen ist.

Daneben kann eine wesentliche und entscheidende berufliche Anforderung im weiteren Sinne gegeben sein. Diese Fälle sind sehr viel schwerer einzukreisen, denn sie sind die eigentlich problematischen Sachverhalte:

Erste Voraussetzung ist hier, dass ein Arbeitnehmer eines bestimmten Geschlechts die berufliche Tätigkeit zwar verrichten kann, jedoch tatsächlich schlechter ausübt als Angehörige des anderen Geschlechts. In einem zweiten Schritt ist dann zu fragen, ob die Minderleistung biologisch, wenn auch uU nicht unmittelbar, sondern reflektiert durch Dritte, mit denen der Arbeitnehmer zu tun hat, bedingt ist. Das ist in allen Fällen gegeben, wo die Scham gegenüber dem anderen Geschlecht relevant wird. Denn bei solchen Sachverhalten (Masseur, ärztliches und medizinisches Personal) sind keine

57 Preis Individualarbeitsrecht, 2. Aufl. 2003, S. 380; Hanau, FS Lüderitz, 2000, S. 247. Ausführlicher s. auch Thüsing, RdA 2001, $319 \mathrm{ff}$.

58 Zur Authentizitätswahrung s. auch Art. R. 123-1 Code du travail; Danach ist eine Ungleichbehandlung wegen des Geschlechts nur bei Schauspielern, Mannequins und Modellen möglich.

59 So bereits vor einer Generation Elias/Napier/Wallington, Labor Law, 1980, S. 53.

60 Z.B. $\S 64$ a BBergG.

61 Das auf verschiedene Gesetze verteilt ist. Für die Geschlechterdiskriminierung erfolgte die Umsetzung garde durch die Ley Orgánica 3/2007, de 22 de marzo, para la igualdad efectiva de mujeres y hombres. 
Vorurteile gegenüber Frauen oder Männern entscheidend, sondern ein Gefühl, das zwar gesellschaftlich geformt sein mag, aber dennoch biologisch begründet ist. Daher ist immer dann, wenn glaubhaft nachgewiesen werden kann, dass Kunden zur Wahrung der Intimsphäre das andere Geschlecht zurückweisen, eine Differenzierung zwischen den Geschlechtern zulässig. Eine Abwägung von Interessen ist nicht erforderlich, denn es wird nur die biologische Verschiedenheit von Mann und Frau anerkannt, nicht aber eine diskriminierende Unterscheidung getroffen. Wie vernünftig oder wie verbreitet das Schamgefühl ist, ist nicht relevant. Entscheidend ist allein, ob dies der tatsächliche Grund für die Differenzierung ist.

In den übrigen Fällen, in denen der Unterschied in der Fähigkeit, einer Aufgabe gerecht zu werden, nicht biologisch begründet ist, ist danach zu fragen, ob nicht dennoch ein legitimes öffentliches Interesse an der bestmöglichen Erfüllung der Aufgabe besteht. Hier ist dann abzuwägen: Zwar mag man die Vorurteile von Häftlingen gegenüber weiblichen oder älteren Bewachern verurteilen, jedoch liegt die Sicherheit des Gefängnisses sicherlich im öffentlichen Interesse und kann daher eine unterschiedliche Behandlung rechtfertigen. ${ }^{62}$

\section{c) Die Entscheidung des Gerichts}

Anders als bei der mittelbaren Diskriminierung ${ }^{63}$ und anderes als bei der Altersdiskriminierung ${ }^{64}$ pflegt der EuGH über die Rechtfertigung einer unmittelbaren Geschlechtsdiskriminierung durch das nationale Recht selber zu entscheiden, wie sich insb. im Verfahren Tanja Kreil gezeigt hat, das zum Recht der Frauen zum Dienst an der Waffe geführt hat. ${ }^{65}$ Auch hier wird es also wohl eine klare Antwort der Luxemburger Richter geben, keinen Rückverweis.

Der dogmatische Kernpunkt der Rechtsfrage liegt darin, dass keiner der dargelegten Rechtfertigungsgründe eingreift, denn anders als bei der Amme gehört das Stillen nicht zu den Dienstpflichten der Arbeitnehmerin, die sich auf die Stillzeit beruft. Was ihre Tätigkeit ist, ist für die Unterscheidung irrelevant; dann kann aber auch kein tätigkeitsbezogener Rechtfertigungsgrund eingreifen. Eine Unterscheidung wäre damit nur dann möglich, wenn sie gar keine unmittelbare Ungleichbehandlung wegen des Geschlechts manifestiert. Das wird man nach der Norm schwer sagen können, da nicht nur das biologische Stillen (das Männer nun mal nicht können), sondern auch das Surrogat Fläschchen mit einbezogen wurde. Wäre das nicht der Fall, wäre die Unterscheidung offensichtlich zulässig, weil beide Geschlechter gleich behandelt würden, so sie denn stillen.

62 S. EuGH v. 11.1.2000 - Rs C-285/98 - Kreil, NJW 2000, 497; siehe auch EuGH v.15.5..1986 - Rs C-222/84 - Johnston, Slg. 1986, 1651, Rnr. 36; EuGH v. 26. 10. 1999, Rs C-273/97 Sirdar, EuZW 2000, 27, Rnr. 23.

63 S. nur EuGH v. 23. 10. 2003 - Rs. C-4/02, Slg. 2003, I-12575.

64 Z.B. EuGH v. 16.10.2007 - Rs. C-411/05, NZA 2007, 1219; hierzu Bauer/Krieger, NJW 2007, 3672; Bayreuther, DB 2007, 2427; Waltermann, ZESAR 2007, 361.

65 EuGH v. 11.1.2000 - Rs C-285/98 - Kreil, NJW 2000, 497; S. hierzu auch Thüsing, Europäisches Arbeitsrecht, 2008, S. 54. 
Da dem aber nicht so ist, spricht viel dafür, dass der EuGH hier eine unzulässige Diskriminierung von Männern annehmen wird. Warten wir ab.

\section{Vorgaben des Arbeitszeitrechts - Vorabentscheidungsersuchen des Tribunale} ordinario di Torino (Italien), eingereicht am 22. Juni 2009 -Antonio Accardo u. a./ Comune di Torino 66

\section{Vorlagefragen:}

Sind die Art. 5, 17 und 18 der Richtlinie 93/104/EG des Rates vom 23. November 1993 über bestimmte Aspekte der Arbeitszeitgestaltung dahin auszulegen, dass sie im innerstaatlichen Recht unabhängig von ihrer förmlichen Umsetzung oder von innerstaatlichen Vorschriften, die ihre Anwendbarkeit auf bestimmte Berufskategorien einschränken, in einem Rechtsstreit, in dem ein Tätigwerden der Sozialpartner im Einklang mit dieser Richtlinie festgestellt wird, unmittelbar anwendbar sind?

Ist das Gericht des Mitgliedstaats unabhängig von einer solchen unmittelbaren Wirkung jedenfalls verpflichtet, eine noch nicht umgesetzte Richtlinie oder, nach ihrer Umsetzung, eine Richtlinie, deren Wirksamkeit durch innerstaatliche Vorschriften ausgeschlossen scheint, als Auslegungsmaßstab und damit als Bezugspunkt für die Behebung möglicher Auslegungszweifel heranzuziehen?

Ist es dem Gericht eines Mitgliedstaats verwehrt, die Unzulässigkeit eines Verhaltens festzustellen und deshalb Schadensersatz zuzusprechen, wenn dieses Verhalten von den Sozialpartnern gestattet worden ist und diese Gestattung mit dem Gemeinschaftsrecht, wenn auch in Form einer nicht umgesetzten Richtlinie, im Einklang steht?

Ist Art. 17 Abs. 3 der Richtlinie 93/104 dahin auszulegen, dass er ein Einschreiten der Sozialpartner und die Einführung einer abweichenden Regelung der wöchentlichen Ruhezeit durch diese in autonomer Weise, d. h. gänzlich losgelöst von Abs. 2 und von der dortigen Aufzählung von Berufen und Tätigkeiten gestattet?

\section{a) Die Vorlage}

Auch das italienische Arbeitszeitrecht ist nicht europarechtskonform. Dies jedenfalls ist die Auffassung das Tribunale ordinario Turin. Man wundert sich, denn Italien war vor gut zwei Jahren eines von nur zwei Ländern, denen die Kommission nicht einen Verstoß

66 Rs C-227/09. 
gegen das europäische Arbeitszeitrecht vorwarf. ${ }^{67}$ Doch wenn auch das italienische Recht nun europarechtswidrig wäre: Was folgt daraus? Der Unterschied zwischen europarechtskonformer Auslegung und direkter Anwendbarkeit der Richtlinie ist auch dem italienischen Juristen bewusst. ${ }^{68}$ Doch auch hier ist Bewegung eingetreten. Nach der Vorlage im Verfahren Kücükdeveci ${ }^{69}$ und den reformfreudigen Anträgen des Generalanwalts Bot zielt die Vorlage des Turiner Gerichts ebenso wie die bereits dargestellte Vorlage des Tribunale di Trani nun in die gleiche Richtung: Inwieweit ist eine unmittelbare Wirkung von Richtlinien auch unter Privaten anzuerkennen, und sei es auch nur in der Fassung, dass der Richtlinie entgegenstehendes nationales Recht nicht angewandt werden soll? Und dann: Welche anderen Sanktionen bestehen?

\section{b) Das europäische Rüstzeug: Unmittelbare Wirkung}

Lange Zeit schien es klar: Die Richtlinie (Art. 249 Satz 3 EG) räumt den Bürgern grundsätzlich keine unmittelbare Rechtsposition ein, sondern verpflichtet nur die Mitgliedstaaten, solche Rechtspositionen zu schaffen. ${ }^{70}$ Hinsichtlich der Wahl der Mittel sind die Mitgliedstaaten frei. Die Handlungsform der Richtlinie entspricht damit eher dem Grundsatz der Subsidiarität, weil größerer Entscheidungsspielraum bei den Mitgliedstaaten verbleibt. ${ }^{71}$ Vom Grundsatz, dass Richtlinien keine unmittelbare Wirkung

67 Nach einer von den Dienststellen der Kommission erstellten Analyse erfolgt die Umsetzung der Richtlinie 2003/88 durch die Mitgliedstaaten in höchst unterschiedlicher Weise. 19 Länder missachten die Vorschriften über den Bereitschaftsdienst (Österreich, Belgien, Zypern, Tschechische Republik, Deutschland, Dänemark, Estland, Griechenland, Spanien, Finnland, Frankreich, Ungarn, Irland, Niederlande, Polen, Schweden, Slowenien, Slowakei, Vereinigtes Königreich). 21 Länder halten sich nicht an die Bestimmungen über Ausgleichsruhezeiten (Belgien, Zypern, Tschechische Republik, Deutschland, Dänemark, Estland, Griechenland, Spanien, Finnland, Frankreich, Ungarn, Irland, Litauen, Lettland, Malta, Niederlande, Portugal, Schweden, Slowenien, Slowakei, Vereinigtes Königreich). 4 Länder verstoßen gegen die Vorschriften für das individuelle Opt-out (Spanien, Frankreich, Ungarn, Vereinigtes Königreich). Lediglich Luxemburg und Italien haben die Arbeitszeitrichtlinie vollständig in nationales Recht umgesetzt - zumindest zum damaligen Zeitpunkt. S. hierzu auch Thüsing, EuZA 2008, 159 ff. Die Umsetzung der Arbeitszeitrichtlinie ist in den verschiedenen Mitgliedsstaaten recht unterschiedlich ausgefallen; lesenswert bereits der Umsetzungsbericht aus dem Jahr 2000, KOM (2000) 787 endg. (http://ec.europa.eu/employment_social/labour_law/ documentation_de.htm\#22).

68 S. zur italienischen Diskussion bereits Thüsing, ZESAR 2009, 182.

69 Hier könnte eine Vorentscheidung im Verfahren Hütter getroffen sein, das einen strukturell vergleichbaren Fall aus Österreich betraf. Der EuGH v. 18.6.2008 (Rs. C-88/08) tenorierte: „Die Art. 1, 2 und 6 der Richtlinie 2000/78/EG des Rates vom 27. November 2000 zur Festlegung eines allgemeinen Rahmens für die Verwirklichung der Gleichbehandlung in Beschäftigung und Beruf sind dahin auszulegen, dass sie einer nationalen Regelung entgegenstehen, die, um die allgemeine Bildung nicht gegenüber der beruflichen Bildung zu benachteiligen und die Eingliederung jugendlicher Lehrlinge in den Arbeitsmarkt zu fördern, bei der Festlegung der Dienstaltersstufe von Vertragsbediensteten des öffentlichen Dienstes eines Mitgliedstaats die Berücksichtigung von vor Vollendung des 18. Lebensjahrs liegenden Dienstzeiten ausschließt"“.

70 Zuletzt BAG v. 24.3.2009 - 9 AZR 983/07, NZA 2009, 538.

71 S. auch EuGH v. 26.10.1993 - Rs. C-163/82, Slg. 1983, 3273. 
gegenüber den Bürgern der Mitgliedstaaten haben, ist nur dann eine Ausnahme zu machen, wenn der Mitgliedstaat die Frist zur Umsetzung der Richtlinie versäumt hat. Enthält die Richtlinie eine zwingende Bestimmung und ist diese hinreichend bestimmt, so dass sie den Betroffenen eine entsprechende Rechtsposition einräumen kann, dann wirkt sie mit Ablauf der Umsetzungsfrist ,,self-executing“. Dennoch trifft es nicht zu, dass die Richtlinie dann in ihrer Rechtswirkung einer Verordnung oder dem unmittelbar geltenden Vertragsrecht gleichkommt, ${ }^{72}$ denn auch dann, wenn die Richtlinie eine unmittelbare Wirkung entfaltet, richtet sie sich allein gegen den Staat, nicht aber gegen andere Bürger. Sie entfaltet also keine horizontale, sondern nur vertikale unmittelbare Wirkung. ${ }^{73}$ Ein Arbeitnehmer kann sich daher nicht gegenüber seinem privaten Arbeitgeber auf die Bestimmung der Richtlinie berufen.

Der wesentliche Grund, warum der EuGH eine unmittelbare horizontale Wirkung von Richtlinien ablehnt, ist der Wortlaut des Art. 249, der eine solche Wirkung eben nur Verordnungen zuweist, ausdrücklich aber nicht Richtlinien. Diese Unterscheidung bleibe auch bei versäumter Umsetzung bestehen. Der EuGH hat in diesem Zusammenhang betont, dass die Annahme einer unmittelbaren horizontalen Wirkung darauf hinauslaufen würde, ,der Gemeinschaft die Befugnis zuzuerkennen, mit unmittelbarer Wirkung zu Lasten der Bürger Verpflichtungen anzuordnen, obwohl sie dies nur dort darf, wo ihr die Befugnis zum Erlass von Verordnungen zugewiesen ist“" 74

Soll dies nun alles anders sein? In sehr gewundenen Anträgen verlangt Bot dies für die Anti-Diskriminierungsrichtlinien, und nun erfragt es das italienische Gericht für die Arbeitszeitrichtlinie. Es geht um die unmittelbare Wirkung von Richtlinien. Eine positive unmittelbare Wirkung in Horizontalverhältnissen, die dazu führt, dass eine Richtlinie als solche wie nationales Recht oder eine EG-Verordnung (Art. 249 Abs. 2 EG) Pflichten für Private begründet, hat der EuGH in ständiger Rechtsprechung abgelehnt. ${ }^{75}$ Eine negative unmittelbare Wirkung oder Ausschlusswirkung führt demgegenüber lediglich dazu, dass nationales Recht, das gegen eine Richtlinie verstößt, unanwendbar ist. Ist sie anzuerkennen? In der Vergangenheit haben sich schon Generalanwälte für eine negative unmittelbare Wirkung in Horizontalverhältnissen ausgesprochen. ${ }^{76}$ Auch ein Teil des Schrifttums befürwortet eine solche Richtlinienwirkung. ${ }^{77}$ Der EuGH hat eine solche Wirkung bisher nicht anerkannt. Die häufig angeführten

72 So aber Krimphove, Europäisches Arbeitsrecht, Rn. 96.

73 So gerade im Arbeitsrecht der EuGH entgegen der Anträge des Generalanwalts Lenz in der Sache Faccini Dori, EuGH v. 14.7.1994 - Rs. C-91/92, Slg. 1994 I, 3325; ebenso GA Mazak in seinen Anträgen in EuGH v. 14.2.2006 - Rs. C-411/05, ABl. Nr. C 36, 20 - Palacios de la Villa.

74 Vgl. EuGH v. 14.7.1994 - Rs. C-91/92 - Faccini Dori, Slg. 1994 I, 3325.

75 S. auch EuGH v. 14.7.1994 - Rs. C-91/92 -Faccini Dori, Slg. 2004, I-3325 Rn 24; v. 7.3.1996 - Rs. C-192/94 -El Corte Inglés, Slg. 1996, I-1281 Rn 17; v. 5.10.2004 - Rs. C-397-403/01 Pfeiffer - NZA 2004, 1145.

76 Vgl. Schlussanträge von GA Saggio vom 16.12.1999 in der Rs. Océano (Rs. C-240-244/98, Slg. 2000, I-4941 Rn 30) und GA Colomer vom 27.4.2004 in der Rs. Pfeiffer (Rs. C-397-403/01, Rn 29 ff.).

77 Herrmann, Richtlinienumsetzung durch die Rechtsprechung, 2003, S. 78 ff. m.w.N. auch aus dem englischsprachigen Schrifttum. 
Entscheidungen CIA Security ${ }^{78}$ und Unilever ${ }^{79}$ lassen sich nicht zwingend als Anerkennung einer horizontalen unmittelbaren Wirkung verstehen. Vielmehr lassen sich die Fälle auf vertikale Konstellationen zurückführen. ${ }^{80}$

c) Das europäische Rüstzeug: Europarechtkonforme Auslegung und ggf. Rechtsfortbildung

Nicht so ganz einleuchtend ist die Frage des Gerichts zur europarechtskonformen Auslegung. Die ist unstreitig anerkannt: Soweit Auslegungsspielraum besteht, haben nationale Gerichte und Behörden das nationale Recht im Lichte der erlassenen, aber noch nicht umgesetzten Richtlinie zu deuten. ${ }^{81}$ Umsetzungslücken können mithin durch ein modifiziertes Verständnis des bereits vorgefundenen nationalen Rechts geschlossen werden. Während also die unmittelbare Wirkung keine Verpflichtung von Privaten bewirken kann, wirkt sich die richtlinienkonforme Auslegung auf sämtliche Rechtsbeziehungen aus. Ihre Grenze findet sie dort, wo Wortlaut, Systematik und Zweck des nationalen Rechts eine Deutung entsprechend der europarechtlichen Vorgaben nicht zulassen: Eine europarechtskonforme Auslegung ist möglich und geboten, eine europarechtskonforme Beugung ist unzulässig. ${ }^{82}$ Hier gilt nichts anderes als für das Gebot der verfassungskonformen Auslegung. ${ }^{83}$ Das gilt dann auch für die Möglichkeiten einer Rechtsfortbildung ${ }^{84}$ - andere Mitgliedstaaten unterscheiden hier ohnehin nicht. Für den französischen Juristen ist das alles interpretation. Ziel der europarechtskonformen Auslegung ist es, einen Widerspruch mit den Vorgaben des Europarechts zu vermeiden. Das BAG formuliert es freilich in jüngster Rechtsprechung deutlich weitergehend im Sinne einer europafreundlichen Auslegung, die sich bereits den Zielen - nicht bloß ihren Mitteln - verpflichtet weiß. ${ }^{85}$

78 EuGH v. 30.4.1996 - Rs. C-194/94, Slg. 1996, I-2201.

79 EuGH v. 26.9.2000 - Rs. C-443/98, Slg. 2000, I-7535.

80 Gundel, EuZW 2001, 143, 147 f.; zum Ganzen auch von Medem, Kündigungsschutz und AGG, 2008, S. $204 \mathrm{ff}$.

81 Grundsätzlich EuGH v. 5.10.2004 - Rs. C-397/01 bis C-403/01- Pfeiffer, NZA 2004, 1135; EuGH v. 10.4.1984 - Rs. C-14/83, Slg. 1984, 1891, 1909; EuGH v. 13.11 .1990 Rs. C-308/89, Slg. 1990 I, 4185.

82 S. BAG v. 18.2.2003 - 1 ABR 2/02 - zu B IV 3 b dd der Gründe, BAGE 105, 32 und EuGH v. 4.7.2006 - Rs. C-212/04 - Adeneler, NJW 2006, 2465; BAG v. 18.2.2003 - 1 ABR 2/02, NZA 2003, 742, 747 im Anschluss an die SIMAP-Entscheidung des EuGH v. 3.10.2000 Rs. C-303/98, Slg. 2000 I, 7963; ebenso entgegen der Schlussanträge des Generalanwalts Colomer in der Rechtssache Pfeiffer, EuGH v. 5.10.2004 - Rs. C-397/01 bis C-403/01, NZA $2004,1135$.

83 S. hierzu BAG v. 18.2.2003 - 1 ABR 2/02, DB 2003, 1387, 1389 mit Anm. Koenigs.

84 Zuletzt - allzu großzügig - BAG v. 24.3.2009 - 9 AZR 983/07, NZA 2009, 538.

85 BAG v. 6.11.2008 -2 AZR 523/07, NZA 2009, 361: „Erfordernis der europarechtskonformen Auslegung des nationalen deutschen Rechts, [wonach] unter mehreren möglichen Auslegungen diejenige den Vorzug erhält, die dem in den Richtlinien des Rates zum Ausdruck gekommenen Ziel eines wirksamen und abschreckenden Schutzes gegen diskriminierende Entlassungen gerecht wird“. 
Bevor die Frist zur Umsetzung abgelaufen ist, besteht nach Auffassung des BAG keine Pflicht zur europarechtskonformen Auslegung. ${ }^{86}$ Dies entspricht nicht der Vorstellung des EuGH: Das Gebot zur richtlinienkonformen Interpretation innerstaatlichen Rechts tritt nach seiner Rechtsprechung nicht erst mit der gezielten Umsetzung des Richtlinieninhalts, sondern bereits mit Ablauf der Umsetzungsfrist ein, ohne dass der Mitgliedstaat die Umsetzung versucht oder in Angriff genommen haben muss. ${ }^{87}$ Hier fragt das Gericht also nach europarechtlichen Selbstverständlichkeiten.

\section{d) Europarechtliches Neuland: Die Rolle der Sozialpartner}

Spannender dann die Frage nach der Rolle der Sozialpartner. ${ }^{88}$ Unterscheiden sich die Sanktionen, wenn nicht der Mitgliedstaat, sondern an seiner Stelle die Sozialpartner die Umsetzung der Richtlinie in die Hand genommen haben. Die Möglichkeit besteht nach Art. 137 Abs. 3 EG. Danach kann der Mitgliedstaat den Sozialpartnern auf deren gemeinsamen Antrag die Durchführung einer Richtlinie übertragen. Hiervon wurde in Deutschland noch nicht Gebrauch gemacht, auch weil Tarifverträge keine allgemeine Verbindlichkeit haben. ${ }^{89}$ Auch wenn der italienische Fall kein solcher ausdrücklicher Umsetzungsfall ist, bleibt die Frage, wieweit Tarifverträge in dieses Sanktionensystem eingebunden sind. Generalanwalt Colomer sagte einmal mutig, auch Tarifverträge seien europarechtskonform auszulegen. ${ }^{90}$ Aber stimmt das? Den Tarifvertragsparteien kann nicht in ähnlicher Weise wie dem Mitgliedstaat unterstellt werden, sie wollten das Europarecht befolgen und mehr noch: zur Wirksamkeit bringen. Und kann es eine unmittelbare Wirkung des Europarechts gegenüber entgegenstehenden Tarifverträgen geben. Wenn Tarifverträge ,kollektiv ausgeübte Privatautonomie“91 sind, dann wohl nicht. Wenn Tarifverträge aber ähnlich wie der Staat an die Grundfreiheiten gebunden sind, ${ }^{92}$ dann wohl eher. Das Europarecht ist hier im Fluss und es bleibt spannend.

86 Im Hinblick auf die Altersdiskriminierung siehe BAG v. 16.8.2005 - 9 AZR 378/04, NZARR 2006, 253.

87 S. GA Tizzano in seinem Schlussantrag zu EuGH v. 22.11.2005 - Rs. C-144/04 - Mangold, Slg. 2005 I, 9981 Rn. 115 unter Hinweis auf die - allerdings nicht eindeutige - Entscheidung des EuGH v. 8.10.1987 - Rs. C-80/86 - Kolpinghuis Nijmegen, Slg. 1987, 3969; s. auch die ausführliche Begründung der GA Kokott, Rs C-212/04 vom 27.10.2005.

88 Informativ auch Deinert, RdA 2004, 211.

89 S. hierzu auch Thüsing, Europäisches Arbeitsrecht, 2008, § 1 Rnr. 78.

90 Schlussanträge v. 27.4.2004- verb. Rs. C-397/01 bis C-403/ 01 - Bernhard Pfeiffer ua / Deutsches Rotes Kreuz. Hiergegen bereits Thüsing, ZIP 2004, 2301.

91 Grundlegend Bayreuther, Tarifautonomie als kollektiv ausgeübte Privatautonomie, 2005; s. bereits; bereits BAG (GS) v. 16.3.1956, AP Nr. 1 zu § 57 BetrVG.

92 S. bereits EuGH v. 8.7.1999 - Rs. C-234/97 - Bobadilla, Slg 1999 I. 4773. 
6. Europäischer Arbeitnehmerbegriff - Vorabentscheidungsersuchen des Augstākās tiesas Senāta (Republik Lettland), eingereicht am 25. Juni 2009-Dita Danosa/LKB Lìzings SIA (Rechtssache C-232/09)

\section{Vorlagefragen}

1. Fallen die Mitglieder des Vorstands von Kapitalgesellschaften unter den gemeinschaftsrechtlichen Begriff des Arbeitnehmers?

2. Stehen Art. 10 der Richtlinie 92/85/EWG ${ }^{93}$ und die Rechtsprechung des Gerichtshofs der Europäischen Gemeinschaften Art. 224 Abs. 4 des Komerclikums entgegen, der die Abberufung von Mitgliedern des Vorstands von Kapitalgesellschaften ohne jede Einschränkung, insbesondere - im Fall einer Frau - ungeachtet des Bestehens einer Schwangerschaft, gestattet?

Ausgangspunkt des Verfahrens ist die Kündigung von Frau Danosa, die hier gegen eine Bank klagt, deren Vorstand sie war. Wie das Verfahren ausgeht, wird sie vielleicht nicht mehr allzu sehr interessieren, da sie erst am 8. Juli in den Vorstand der lettischen Post bestellt wurde. Weil sie aber auch zurzeit Jurastudentin an der Universität Riga ist, ist es vielleicht auch nur das juristische Interesse, das sie diesen Prozess weiterverfolgen lässt.

Was fragt nun das Gericht? Die Frage ist nicht allzu klug gestellt. Wer pauschal nach den Vorstandsmitgliedern von Kapitalgesellschaften fragt, wird keine allzu hilfreiche Antwort bekommen können. Denn hier ist nach den verschiedenen Rechtsordnungen zu differenzieren, wie auch nach den verschiedenen Gesellschaftsformen zu differenzieren ist. Hier zeigt exemplarisch das deutsche Recht ein differenziertes Bild:

Arbeitnehmer ist nach dem Verständnis des deutschen Arbeitsrechts, wer weisungsgebunden Dienste für einen anderen erbringt. Wer im Wesentlichen frei seine Tätigkeit und seine Arbeitszeit bestimmen kann, ist selbständig. ${ }^{94}$ Ist die Weisungsunterworfenheit damit der Dreh- und Angelpunkt der Qualifikation von Organmitgliedern als Arbeitnehmer, folgt daraus, dass GmbH-Geschäftsführer grundsätzlich als Arbeitnehmer angesehen werden können, ${ }^{95}$ da sie einem arbeitsrechtlichen Weisungsrecht unterworfen sein können (dieses ist zu trennen vom gesellschaftsrechtlichen Weisungsrecht nach $\S 37$ Abs. 1 GmbHG). ${ }^{96}$ Demgegenüber sind Vorstände einer Aktiengesellschaft keine Arbeitnehmer, ${ }^{97}$ da sie weisungsunabhängig sind ( $\$ 76$ Abs. 1 AktG). Die Überwachung durch den Aufsichtsrat macht sie nicht zu Arbeitnehmern, da dieser keine konkreten Einzelweisungen erteilen kann. ${ }^{98}$ Noch ungeklärt ist der Einfluss des Verhältnisses der

93 Richtlinie 92/85/EWG des Rates vom 19. Oktober 1992 über die Durchführung von Maßnahmen zur Verbesserung der Sicherheit und des Gesundheitsschutzes von schwangeren Arbeitnehmerinnen, Wöchnerinnen und stillenden Arbeitnehmerinnen am Arbeitsplatz (zehnte Einzelrichtlinie im Sinne des Artikels 16 Absatz 1 der Richtlinie 89/391/EWG), ABl. Nr. L-348 v. 28.11.1992, S. $1 \mathrm{ff}$.

94 BAG v. 27.3.1991 - 5 AZR 194/90, NZA 1991. 933.

95 BAG v. 26.5.1999 - 5 AZR 664/98, NJW 1999, 3731; Köhl, DB 1996, 1597.

96 BAG v. 26.5.1999 - 5 AZR 664/98, NJW 1999, 3731.

97 Thüsing, in: Fleischer, Handbuch des Vorstandsrechts, § 4 Rn. 54 f.

98 Mankowski, RIW 2004, 167, 169. 
geschäftsführenden Direktoren zu den nicht geschäftsführenden Direktoren in der monistisch verfassten SE auf den Arbeitnehmerstatus der Erstgenannten. ${ }^{99}$

So viel zur Rechtslage in Deutschland. Im Gemeinschaftsrecht ist der Arbeitnehmerbegriff jedoch nicht definiert, es existiert keine für das EG-Recht allgemeingültige Definition. ${ }^{100}$ Der Arbeitnehmerbegriff ist vielmehr im jeweiligen Regelungszusammenhang auszulegen. ${ }^{101}$ Im Rahmen des Art. 39 EGV gilt der arbeitsrechtliche Arbeitnehmerbegriff, der vom EuGH unter Zustimmung der Literatur definiert ist. ${ }^{102}$ Wesentliches Kriterium des Arbeitsverhältnisses ist danach auch im Gemeinschaftsrecht die Weisungsgebundenheit des Arbeitsnehmers. ${ }^{103}$ Eine Tätigkeit nach Weisungen liegt dann vor, wenn eine Leistung für fremde Rechnung und auf fremdes Risiko erbracht wird. Es bestehen also insoweit keine Unterschiede zwischen dem gemeinschaftlichen und dem deutschen Arbeitnehmerbegriff. ${ }^{104}$ Der Begriff des unselbstständigen Erwerbstätigen ist im Gemeinschaftsrecht hingegen weiter gefasst als der des Arbeitnehmers. Abzustellen ist insoweit auf den Arbeitnehmerbegriff des Art. 48 EG-Vertrag. Gemeinschaftsrechtlich ist etwa der Geschäftsführer als Träger der Niederlassungsfreiheit, nicht aber der Arbeitnehmerfreizügigkeit dann anzusehen, wenn er nach der gesetzlichen oder satzungsmäßigen Verteilung der Befugnisse in der juristischen Person der Weisung keiner anderen Person oder keines Organs unterliegt, das er selbst nicht kontrolliert. ${ }^{105}$ Damit ist der Geschäftsführer einer deutschen $\mathrm{GmbH}$ regelmäßig wohl auch Arbeitnehmer i.S. des Gemeinschaftsrechts, sofern er einem arbeitsrechtlichen Weisungsrecht unterworfen ist. ${ }^{106}$

Fragt das vorlegende Gericht nun spezifisch nach den Besonderheiten des lettischen Rechts? In diesem Fall ist daran zu erinnern, dass der EuGH in ständiger Rechtsprechung darauf hinweist, dass es erforderlich ist, dass das vorlegende Gericht den tatsächlichen und rechtlichen Rahmen, in den sich die von ihm gestellten Fragen einfügen, festlegt oder zumindest die tatsächlichen Annahmen erläutert, auf denen diese Fragen beruhen, um zu einer dem nationalen Gericht sachdienlichen Auslegung des Gemeinschaftsrechts

99 Teichmann, in: Lutter/Hommelhoff, SE-Kommentar, Anh. Art. 43 SE-VO (§ 40 SEAG) Rn. 5: „Sie stehen [...] dem Geschäftsführer einer GmbH wesentlich näher als dem Vorstand einer deutschen Aktiengesellschaft.“.

100 Griebeling NZA 1998, 1137, 1142; Hanau/Steinmeyer/Wank, Handbuch des europäischen Arbeits- und Sozialrechts, 2002, § 14 Rn. 1.

101 EuGH v. 23.3.1982 - Rs. 53/81 - Levin, Slg. 1982, 1035, Rn. 9; v. 11.7.1985 - Rs. 105/84 - Mikkelsen, Slg. 1985, 2639, Rn. 23.

102 EuGH v. 19.3.1964 - Rs. 75/63 - Unger, Slg. 1964, 381, 396 f.; v. 23.3 .1982 - Rs. 53/81Levin, Slg. 1982, 1035, Rn. 23; Hanau/Steinmeyer/Wank, Handbuch des europäischen Arbeits- und Sozialrechts, 2002, § 14 Rn.5 m.w.N.

103 EuGH v.3.7.1986 - Rs. 66/85 - Lawrie-Blum, Slg. 1986, 2121, Rn. 17; v. 26.2.1992 Rs C-357/89 - Raulin, Slg. 1992, 1054, Rn. 10.

104 S. dazu Griebeling NZA 1998, 1137, 1142.

105 GA Leger, Schlussanträge vom 15.2.1996 in der Rs. C-107/94, Slg. 1996, I-3091, 3096; Mankowski, RIW 2004, 167, 169.

106 S. nur Streinz/Franzen EU/EGV Art. 39 EG RdNr. 21; vgl. auch EuGH Slg. 1998, I-2521, I-2547 Rn. 30; Wank in: Hanau/Steinmeyer/Wank, Handbuch europäisches Arbeits- und Sozialrecht, 2003, § 14 RdNr. 14; s. auch Thüsing NZA 2004, Beilage Heft 22 S. 1, 4. 
zu gelangen. ${ }^{107} \mathrm{Ob}$ das geschehen ist, ist mir nicht bekannt, da die Vorlage nur in lettisch veröffentlicht ist. Allerdings kennt auch das lettische Recht eine ähnliche Einteilung nach Gesellschaftsformen wie das deutsche Recht, nämlich die akciju sabiedrība (vergleichbar der Aktiengesellschaft) einerseits und die sabiedrība ar ierobežotu atbildību (vergleichbar der $\mathrm{GmbH}$ ) andererseits. Sollte auch die Stellung der Organe dieser Gesellschaften ihren jeweiligen deutschen Pendants entsprechen, wäre die erste Vorlagefrage äußert ungeschickt formuliert. Der Gerichtshof könnte dies zum Anlass nehmen, das Gebot vertrauensvoller Zusammenarbeit zwischen vorlegendem Gericht und Gerichtshof weiter zu präzisieren. ${ }^{108}$

Bislang wendet die deutsche Literatur $\S 9 \mathrm{MuSchG}$ nicht auf $\mathrm{GmbH}-\mathrm{Geschäftsfüh-}$ rerinnen an. ${ }^{109}$ Das $\mathrm{BAG}^{110}$ hatte sich zwar bereits vor einer Dekade mit der Frage zu befassen, ob der Kündigung einer GmbH-Geschäftsführerin $\S 9$ Abs. 1 MuSchG entgegenstehen könne, wich einer Stellungnahme jedoch aus, da die Klägerin nicht als Arbeitnehmerin zu qualifizieren sei. Dieses Meinungsbild könnte sich ändern, wenn der EuGH seine Rechtsprechung verdeutlicht. Bekanntlich orientiert sich der EuGH bei der Auslegung des Gemeinschaftsrechts vornehmlich an den vom Gesetzgeber verfolgten Zwecken, misst dem teleologischen Argument also besonderes Gewicht bei. ${ }^{111}$ Der Arbeitnehmerbegriff wird wie gesehen ebenfalls anhand dieser Zwecke von Rechtsgebiet zu Rechtsgebiet unterschiedlich beurteilt. Zweck der Richtlinie 92/85/EWG ist der Schutz von schwangeren Arbeitnehmerinnen gegenüber gefährlichen Agenzien, Verfahren oder Arbeitsbedingungen (Erwägungsgrund 11 Richtlinie 92/85/EWG). Schwangere und vor allem der empfindliche nasciturus bedürfen besonderen Schutzes vor schädlichen Einwirkungen. Als Arbeitnehmerinnen sind Schwangere aber der Gefahr ausgesetzt, kraft des Weisungsrechts des Arbeitgebers gezwungen zu sein, solche Gefahren auf sich nehmen zu müssen. Um ihnen aus dieser Zwickmühle zu helfen, gibt es die Richtlinie 92/85/EWG. Auch diese stellt also insbesondere auf die Weisungsgebundenheit der Schwangeren ab. Diese freilich ist eine Frage des Einzelfalls, wie sich in der Rechtsprechung des BAG schon gezeigt hat. Statt also pauschal „Organmitglieder" vom Anwendungsbereich des $\S 9$ Abs. 1 MuSchG auszunehmen, wird man möglicherweise auch in der deutschen Literatur künftig stärker nach den Gesellschaftsformen und den Umständen des Einzelfalls zu differenzieren haben. Man darf das Urteil des EuGH mit Spannung erwarten.

107 EuGH v. 16.7.1992, Rs. C-83/91, DB 1992, 1667; EuGH 26.1.1993, Rs. C-320/90 und C-322/90 - Telemarsicabruzzo, Slg. 1993, I-393 Rn. 5 f.; Callies/Ruffert/Wegener, EGV/ EUV, Art. 234 EG Rn. 33.

108 Dazu etwa EuGH v. 16.7.1992, Rs. C-83/91 - Meilicke, DB 1992, 1667.

109 ErfK/Schlachter, § 1 MuSchG Rn. 3 („Organmitglieder"); HWK/Hergenröder, § 1 MuSchG Rn. 4 („Organmitglieder"); Keil, EWiR 2000, 69.

110 NZA 1999, $987 \mathrm{f}$.

111 Pernice/Mayer, in: Grabitz/Hilf, EUV/EGV, Art. 220 EG Rn. 46; Riesenhuber, in: Riesenhuber, $\S 11$ Rn. 42; s. z.B. EuGH v. 29.11.1956, Rs. 8/55, Slg. 1955/56, 197, 312 -Fédération Carbonnière de Belgique; v. 17.1.1980, Rs. 792/79 - Camera care, Slg. 1980, 119, 129 f., 131; v. 6.10.1970, Rs. 9/70, Slg. 1970, 825, 838; v. 23.3.2006, Rs. C-465/04 Grad, Slg. 2006, I-2879 Rn. $16-22,26$ f. 
7. Folgen des Betriebsübergangs für LeiharbeitnehmerVorabentscheidungsersuchen des Gerechtshof te Amsterdam (Niederlande), eingereicht am 3. Juli 2009 - Albron Catering B.V./FNV Bondgenoten, John Roest (Rechtssache C-242/09)

Vorlagefragen:

Ist die Richtlinie 2001/23/EG ${ }^{1}$ in dem Sinn auszulegen, dass es sich nach Art. 3 Abs. 1 Satz 1 nur dann um einen Übergang von Rechten und Pflichten auf den Erwerber handelt, wenn der Veräußerer des zu übertragenden Unternehmens auch der formelle Arbeitgeber der betroffenen Arbeitnehmer ist, oder bringt der mit der Richtlinie beabsichtigte Schutz der Arbeitnehmer mit sich, dass bei einem Übergang eines Unternehmens einer zu einem Konzern gehörenden Betriebsgesellschaft die Rechte und Pflichten in Bezug auf die für dieses Unternehmen tätigen Arbeitnehmer auf den Erwerber übergehen, wenn das gesamte innerhalb des Konzerns tätige Personal bei einer (ebenfalls zu diesem Konzern gehörenden) Personalgesellschaft beschäftigt ist, die als zentraler Arbeitgeber fungiert?

Wie lautet die Antwort auf den zweiten Teil der ersten Frage, wenn die dort genannten Arbeitnehmer, die für ein zu einem Konzern gehörendes Unternehmen arbeiten, bei einer anderen, ebenfalls zu diesem Konzern gehörenden Gesellschaft beschäftigt sind, die keine Personalgesellschaft ist, wie sie in der ersten Frage beschrieben wurde?

Die Vorlagefrage trifft auf einen weißen Fleck in der Rechtsprechung zum Betriebsübergang: Gehen auch Leiharbeitnehmer über, wenn der Betrieb, in dem sie beschäftigt sind, veräußert wird? ${ }^{12}$ Die Frage des Amsterdamer Gerichtshofs bezieht sich zwar allein auf konzernangehörige Gesellschaften und damit regelmäßig auf die echte Leiharbeit, aber die Frage hat über diese Grenzen hinaus Bedeutung auch für die Arbeitnehmerüberlassung insgesamt.

Nach deutschem Recht würde man einen Übergang der Leiharbeitnehmer intuitiv verneinen, denn die Leiharbeitnehmer stehen gerade nicht in einer arbeitsvertraglichen Beziehung zum Inhaber des entleihenden Betriebs. Vielleicht ist das der Grund, warum diese Frage im deutschen Schrifttum bislang kaum diskutiert wurde. ${ }^{113}$ Doch der Wortlaut der Richtlinie 2001/23/EG stellt auf die vertraglichen Beziehungen zum Betriebsinhaber nicht ab. Nach Art. 3 Abs. 1 RL gehen die Rechte und Pflichte des Veräußerers aus einem Zeitpunkt des Übergangs bestehenden Arbeitsvertrag „oder Arbeitsverhältnis“" aufgrund des Übergangs auf den Erwerber über. Der Begriff des Arbeitsverhältnisses kann hier weiter sein als der des Arbeitsvertrags und u.U. eben nicht auf die vertragliche Verbindung abstellen. Auf die Richtlinie 2008/104/EG könnte sich eine solche Auslegung zwar nicht stützten (hier fehlen entsprechende Anhaltspunkte), doch bliebe gerade in dem vom vorlegenden Gericht angesprochenen Fall ein unbefriedigendes Gefühl im Fall eines engeren Verständnisses: In dem Moment, wo ein Konzern alle seine Arbeitnehmer in einer Personalführungsgesellschaft bündelt, könnte jeglicher Betrieb und jegliches Unternehmen ohne Arbeitnehmer veräußert werden und man würde in einem zweiten Schritt die an die betreffenden Konzernteile überlassenen Arbeitneh-

112 Zum Übergang von Leiharbeitnehmern nicht als Folge, sondern als Voraussetzung eines Betriebübergangs s. EuGH v. 13.9.2007 - C-458/05 - Jouini u.a., NJW 2007, 3195.

113 Leiharbeitnehmer gehen nicht über: A/P/S/Steffan, §613 a BGB Rn. 82; MüKo/MüllerGlöge, § 613 a BGB Rn. 80; Staudinger/Annu $\beta$, § 613 a BGB Rn 28. 
mer problemlos betriebsbedingt kündigen. Das Ziel, das die Betriebsübergangsrichtlinie prominent in Abschnitt 3 ihrer Erwägungen zusammenfasst, wird so konterkariert. Der effet utile könnte ein naheliegendes argumentatives Vehikel sein, die Grenze des Wortlauts der Richtlinie zu überschreiten.

Doch reicht das? Vieles spricht in dieser Richtlinie dagegen. Nach Art. 2 Abs. 2 lässt die Richtlinie das einzelstaatliche Recht im Bezug auf die Begriffsbestimmung des Arbeitsvertrags oder des Arbeitsverhältnisses unberührt - und nach deutschem Recht steht der Arbeitnehmer eben nicht in einem Arbeitsverhältnis zum Entleiher. Zwar heißt es weiterhin, dass die Mitgliedstaaten vom Anwendungsbereich der Richtlinie Arbeitsverhältnisse nicht allein deshalb ausschließen dürfen, weil es sich um Leiharbeitsverhältnisse handelt und bei dem übertragenen Unternehmen um Verleihunternehmen. Dies betrifft jedoch nur den umgekehrten Fall, dass Betriebsteile eines Verleihunternehmens übertragen werden, nicht jedoch den Fall, dass Betriebsteile eines Entleiherunternehmens den Inhaber wechseln.

Es bleibt abzuwarten, ob der EuGH hier ein klares Ja oder Nein antwortet oder einen vermittelnden Weg sucht und sich - der Sache nach nicht fern liegend - vielleicht auf das Rechtsinstitut des Rechtsmissbrauchs beruft, dem auch das europäische Recht keinen Vorschub leisten darf. ${ }^{114}$

\section{Und noch einmal Altersgrenzen: Vorabentscheidungsersuchen des Rayonen sad Plovdiv (Bulgarien), eingereicht am 6. Juli 2009 - Vasil Ivanov Georgiev/ \\ Tehnicheski universitet - Sofia, Filial Plovdiv (Rechtssache C-250/09)}

\section{Vorlagefragen:}

Stehen die Bestimmungen der Richtlinie 2000/78/EG des Rates vom 27. November 2000 zur Festlegung eines allgemeinen Rahmens für die Verwirklichung der Gleichbehandlung in Beschäftigung und Beruf der Anwendung eines nationalen Gesetzes entgegen, das den Abschluss unbefristeter Arbeitsverträge mit Professoren, die das 65. Lebensjahr vollendet haben, nicht zulässt? Konkreter, sind unter Berücksichtigung von Art. 6 Abs. 1 der Richtlinie die in Art. 7 Abs. 1 Nr. 6 des Gesetzes zum Schutz vor Diskriminierung genannten Maßnahmen, die Altergrenzen für die Beschäftigung auf einer konkreten Stelle einführen, objektiv und angemessen und durch ein legitimes Ziel gerechtfertigt sowie verhältnismäßig, unter Beachtung dessen, dass die Richtlinie in vollem Umfang in bulgarisches Recht umgesetzt worden ist?

Stehen die Bestimmungen der Richtlinie 2000/78/EG des Rates vom 27. November 2000 zur Festlegung eines allgemeinen Rahmens für die Verwirklichung der Gleichbehandlung in Beschäftigung und Beruf der Anwendung eines nationalen Gesetzes entgegen, wonach Professoren, die das 68. Lebensjahr vollendet haben, zwangsweise in den Ruhestand versetzt werden? Ist es angesichts der dargelegten Tatsachen und Umstände der vorliegenden Rechtssache und im Fall der Feststellung eines Widerspruchs zwischen den Bestimmungen der Richtlinie und dem einschlägigen nationalen Recht, in das die Richtlinie umgesetzt wurde, möglich, dass die Auslegung der Bestimmungen des Gemeinschaftsrechts zur Nichtanwendung des nationalen Rechts führt?

114 Zum abus de droit aus europäischer Sicht s. umfassend Fleischer, JZ 2003, 865. 
Wieder mal betrifft die Vorlage Altersgrenzen nach mitgliedstaatlichem, diesmal nach bulgarischem Recht. Es handelt sich um die zweite bulgarische Vorlage an den EuGH überhaupt. Im Kontext der Altersgrenzen ist sie nun nach Mangold, ${ }^{115}$ Palacios, ${ }^{116} \mathrm{Kü-}$ cükdeveci, ${ }^{117}$ Wolf $^{118}$ und Petersen ${ }^{119}$ die nächste Gelegenheit für den EuGH, zu den Grenzen der Rechtfertigung einer Benachteiligung wegen des Alters Stellung zu nehmen. Für den Universitätslehrer sympathisch ist, dass Verfahrensgegenstand gerade die Altersgrenze der Professoren ist. Rechtsvergleichend sticht hier die Entwicklung des US-amerikanischen Rechts ins Auge. Als der Age Discrimination in Employment Act 1967 geschaffen wurde, erhielt er auch eine ausdrückliche Ausnahme des Diskriminierungsverbots für Universitätsprofessoren in Lebenszeitanstellung, die zumindest mit 70 Jahren zulässig sein sollte (ADEA 1986 Section 12 (d)). Diese Höchstgrenze fiel 1993 weg. Seitdem können Professoren sich tatsächlich vollbesoldet bis an ihr Lebensende Wissenschaft und Lehre widmen. Die Konsequenzen für die Entwicklung der Universitäten werden in den USA kontrovers diskutiert. ${ }^{120}$

In der Tat leuchtet es hier weniger als bei Ärzten und Piloten ein, dass ein bestimmtes Alter zum Hilfskriterium nachlassender beruflicher Fähigkeiten gewählt wird. Eine differenzierende Antwort des EuGH könnte Folgen auch für das deutsche Recht haben. Nach der Föderalismusreform kann die gesetzliche Altersgrenze nun länderspezifisch geregelt werden. Beamtete Professoren treten zwar - noch immer - regelmäßig mit Ablauf des Monats in den Ruhestand, in dem sie das 65. Lebensjahr vollendet haben. Einige Bundesländer aber weichen von dieser Altersgrenze bereits heute ab. Jüngstes Beispiel einer fließenden Entwicklung ist hier die Änderung des Beamtenrechts Nordrhein-Westfalen, die zu einer Anhebung der Regelaltersgrenze für Professoren geführt hat. ${ }^{121}$ Im Schlepptau nicht unbeeinflusst von diesen Entwicklungen befinden sich instanzgerichtliche Entscheidungen. Über die Revision einer Entscheidung des Landesarbeitsgerichts Köln vom 12.2.2009 ${ }^{122}$ wird das Bundesarbeitsgericht demnächst zu entscheiden haben. Das Kölner Gericht entschied, dass die Altersgrenze für Nachwuchswissenschaftler unwirksam ist. Hier wird es schwer sein, starre Altersgrenzen zu rechtfertigen, so dass die Antwort des EuGH mit Spannung erwartet werden kann.

115 EuGH v. 22.11.2005 - Rs. C-144/04, ZESAR 2006, 65.

116 EuGH v. 16.10.2007 - Rs. C-411/05, ZESAR 2008, 42.

117 Bislang liegen lediglich die Schlussanträge des GA Bot v. 11.3.2008 - Rs. C-555/07, ABl. EU 2008, Nr. C 79, 12 vor.

118 Noch anhängiges Vorlageverfahren, Rs. C-229/08, ABl. EU 2008, Nr. C 223, 21.

119 Noch anhängiges Vorlageverfahren, Rs C-341/08, AB1. EU 2008, Nr. C 260, 8.

120 S. hierzu Hammond/Morgan, Ending Mandatory Retirement for Tenured Faculty, 1991.

121 S. § 31 Abs. 2 LBG NW in der Neufassung durch das Gesetz zur Änderung dienstrechtlicher Vorschriften vom 21.4.2009 GV NRW, S. 224.

1227 SA 1132/08, n.v. (juris). 
9. Sanktionen der Diskriminierung: Vorabentscheidungsersuchen des

Landesarbeitsgerichts Hamburg (Deutschland) eingereicht am 6. Juli 2009 -

Susanne Bulicke gegen Deutsche Büroservice GmbH (Rechtssache C-246/09)

Vorlagefrage:

Verstößt eine nationale Gesetzgebung, nach der (außerhalb von kollektivrechtlichen Regelungen) zur schriftlichen Geltendmachung eines Schadens- und/oder Entschädigungsanspruches wegen Diskriminierung bei der Einstellung eine Frist von zwei Monaten nach Empfang der Ablehnung - oder im Wege der Auslegung: nach Kenntnis der Diskriminierung - gilt, gegen Primärrecht der EG (Gewährleistung eines effektiven Rechtsschutzes) und/oder das gemeinschaftsrechtliche Verbot der Altersdiskriminierung, Richtlinie 2000/78/EG vom 27. November 2000, wenn für gleichwertige Ansprüche nach nationalem Recht dreijährige Verjährungsfristen gelten und/oder das Verschlechterungsverbot gemäß Art. 8 der Richtlinie 2000/78/EG, wenn eine frühere nationale Vorschrift bei der Diskriminierung wegen des Geschlechts eine längere Ausschlussfrist vorsah?

Die Frage ist bereits mehrfach im deutschen Schrifttum aufgeworfen worden, Gerichte haben sich ihr gestellt ${ }^{123}$ und auch die Kommission hat bereits ein Vertragsverletzungsverfahren eingeleitet, weil es davon ausgeht, dass hier die Diskriminierungsrichtlinien nicht hinreichend umgesetzt wurden. ${ }^{124}$ So rügt sie, dass die Frist des $\S 15$ Abs. 4 AGG bereits anläuft, wenn der Bewerber von den diskriminierenden Umständen keine Kenntnis bekommen hat. Dies entspricht aber bereits der alten Rechtslage des $\S 611$ a Abs. 4 BGB, ohne dass dies je vom EuGH beanstandet worden wäre. Dennoch: In der Literatur wird die Zwei-Monats-Regelung des § 15 Abs. 4 AGG von vielen Stimmen bereits unter dem Gesichtspunkt der Gleichwertigkeit für gemeinschaftswidrig erachtet, zum Teil wird auf die Notwendigkeit der allgemeinen Verjährungsfrist von drei Jahren in $\S 195$ BGB abgestellt, zum Teil auf andere Fristen. ${ }^{125}$ Auch gibt es Stimmen, die auf das Verschlechterungsverbot hinweisen. Die Vorschrift dürfte - so das LAG Hamburg - auch aus diesem Grunde gemeinschaftsrechtswidrig sein. ${ }^{126}$

Das Ganze überzeugt nicht so recht. Das BAG hat jüngst noch seine Entscheidung auf $\S 15$ Abs. 4 AGG gestützt, ohne dem EuGH die Frage der Europarechtskonformität vorzulegen. ${ }^{127}$ Warum auch? Das Gesetz ist hier klar, und die Richtlinien wirken nicht unmittelbar unter Privaten. Wer hier nicht - wie Generalanwalt Bot ${ }^{128}$ - neue Wege beschreiten will, der darf wegen fehlender Entscheidungserheblichkeit nicht vorlegen. Das gilt aber auch der Sache nach: Das Verschlechterungsverbot hat eine nur sehr eingeschränkte Bedeutung, wie bereits - wie dargestellt - Generalanwältin Kokott, aber auch Generalanwalt Tizzano in seinen Schlussanträgen im Verfahren Mangold deutlich

123 S. LAG München, 5 Sa 385/08, das selber europarechtskonform auslegte, ohne vorzulegen, in dem Sinne, das die Frist erst ab Kenntnis des Arbeitnehmers von der Diskriminierung laufen könne.

124 Zum Vertragsverletzungsverfahren s. Thüsing, RdA 2008, 317.

125 Rust/Falke, AGG. Nr. $50 \mathrm{zu} \S 15$; Wendeling-Schröder aaO; ErfK aaO. Nr. 12 zu $\S 15$ AGG.

126 Rust/Falke, AGG. Nr. $50 \mathrm{zu} \S 15$.

127 V. 24.9.2009 - 8 AZR 705/08.

128 Schlussanträge v. 11.3.2008 - Rs. C-555/07 - Kücükdeveci, ABl. EU 2008, Nr. C 79, 12, s. hierzu bereits unter II 3 der Darstellung. 
gemacht hat: Es „handelt es sich ... um eine Transparenzklausel, d. h. eine Klausel, die es, um Missbräuche zu verhindern, den Mitgliedstaaten verbietet, die Umsetzung der Richtlinie zum Anlass zu nehmen, um in einem sensiblen Bereich wie dem der Sozialpolitik einen Abbau des in ihrer eigenen Rechtsordnung bereits gewährleisteten Schutzes vorzunehmen und diesen dann (was leider öfter vorkommt!) statt einer unabhängigen Entscheidung auf nationaler Ebene nicht existierenden Gemeinschaftsverpflichtungen zuzuschreiben.“. ${ }^{129}$ Es geht um das Verbot der Rechtfertigung einer Absenkung mit der Umsetzung. Überzeugender ist da der Hinweis auf das Gebot der Effektivität der Sanktionen. Der Grundsatz der Effektivität besagt - im Zusammenhang mit Ausschlussfristen -, dass diese nicht so ausgestaltet sein dürfen, dass sie es praktisch unmöglich machen oder übermäßig erschweren, die Entschädigung zu erlangen. ${ }^{130}$ Hier kann es in der Tat geboten sein, das der Anknüpfungspunkt das Kennen oder Kennenmüssen der Benachteiligung ist. Aber gerade das sieht $\S 15$ Abs. 4 AGG für den Regelfall vor. Einzig in der Bewerbungssituation genügt zum Ingangsetzen der Frist die Ablehnung der Bewerbung. Auch dies aber ist zumutbar: Der Bewerber weiß dann, dass er handeln muss, will er seine Rechte wahren. Nichts anderes gilt etwa im Bereich des Kündigungsschutzes, dessen noch kürzere Dreiwochenfrist auch nicht an der Kenntnis der Kündigungsgründe, sondern am Zugang der Kündigung festmacht. Es fällt schwer zu argumentieren, das deutsche Kündigungsrecht sei deshalb nicht effektiv. Gleiches gilt hier.

129 Anträge vom 30.6.2005, C-144/04.

130 Vgl. etwa EuGH vom 10.7.1997 - Rs. C-261/95 - Palmisani zu Nr. 27, NZA 1997, S. 1041. 